\title{
THE LANCET
}

\section{Supplementary webappendix}

This webappendix formed part of the original submission and has been peer reviewed. We post it as supplied by the authors.

Supplement to: Prospective Studies Collaboration. Body-mass index and cause-specific mortality in 900000 adults: collaborative analyses of 57 prospective studies. Lancet 2009; published online March 18. DOI:10.1016/S0140-6736(09)60318-4. 
Webfigure 1: All-cause mortality vs. BMI in the range $15-50 \mathrm{~kg} / \mathrm{m}^{2}$, by age at risk (excluding the first 5 years of follow-up)

Conventions as in figure 2.

(a) Male

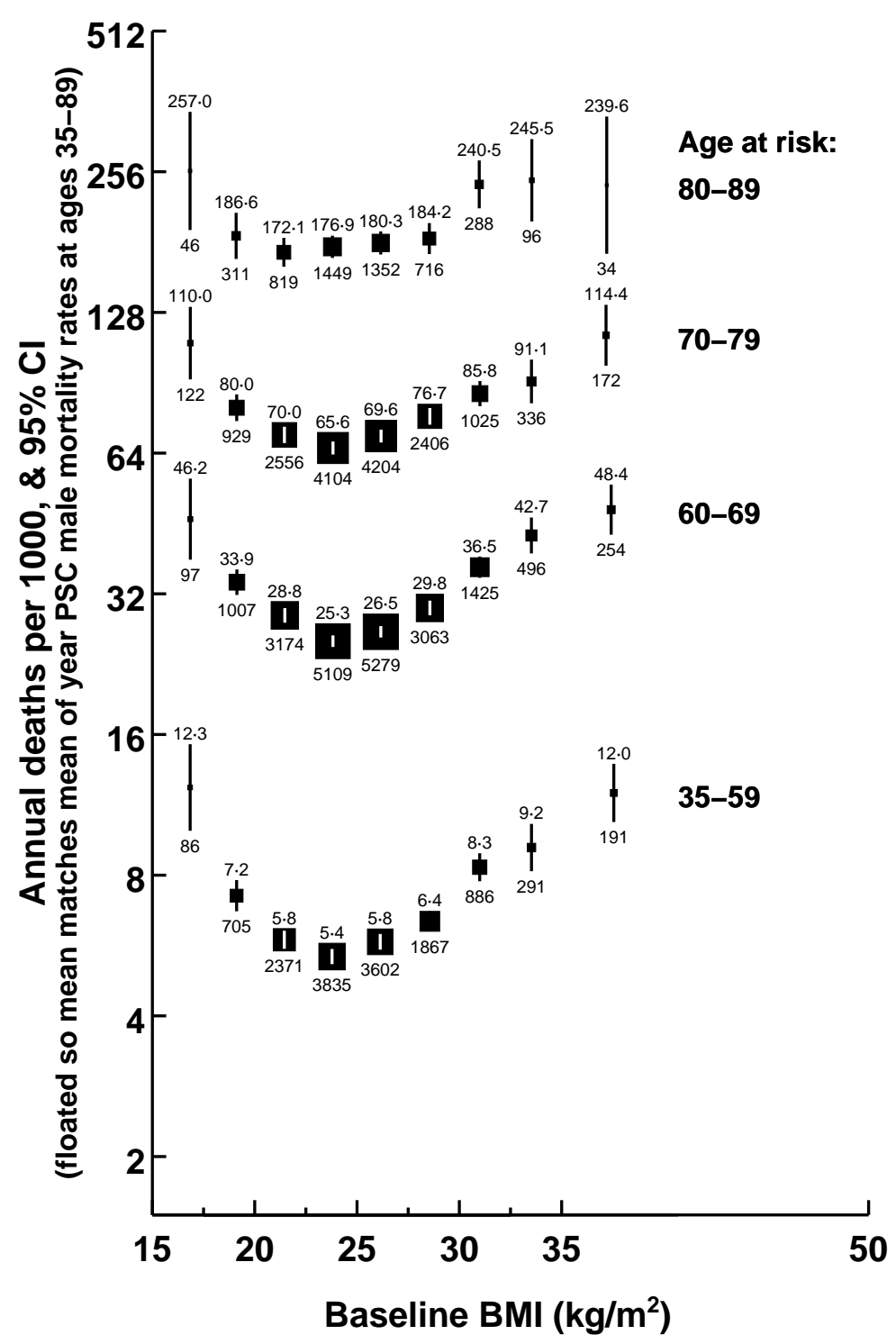

(b) Female

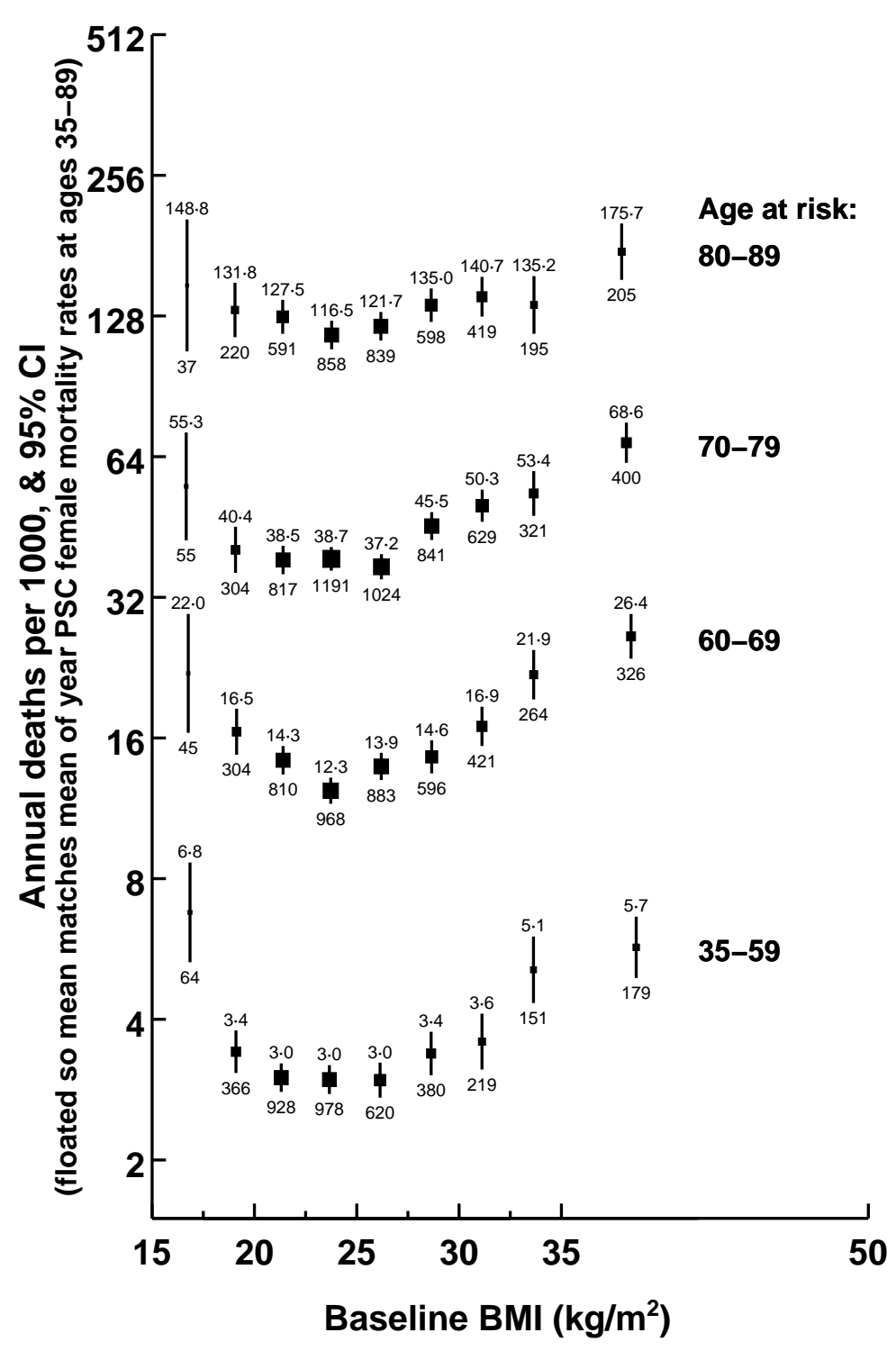


Webfigure 2: IHD mortality vs. BMI for never-smokers in the range $15-50 \mathrm{~kg} / \mathrm{m}^{2}$ (excluding the first 5 years of follow-up)

Conventions as in figure 2, except not adjusted for smoking.

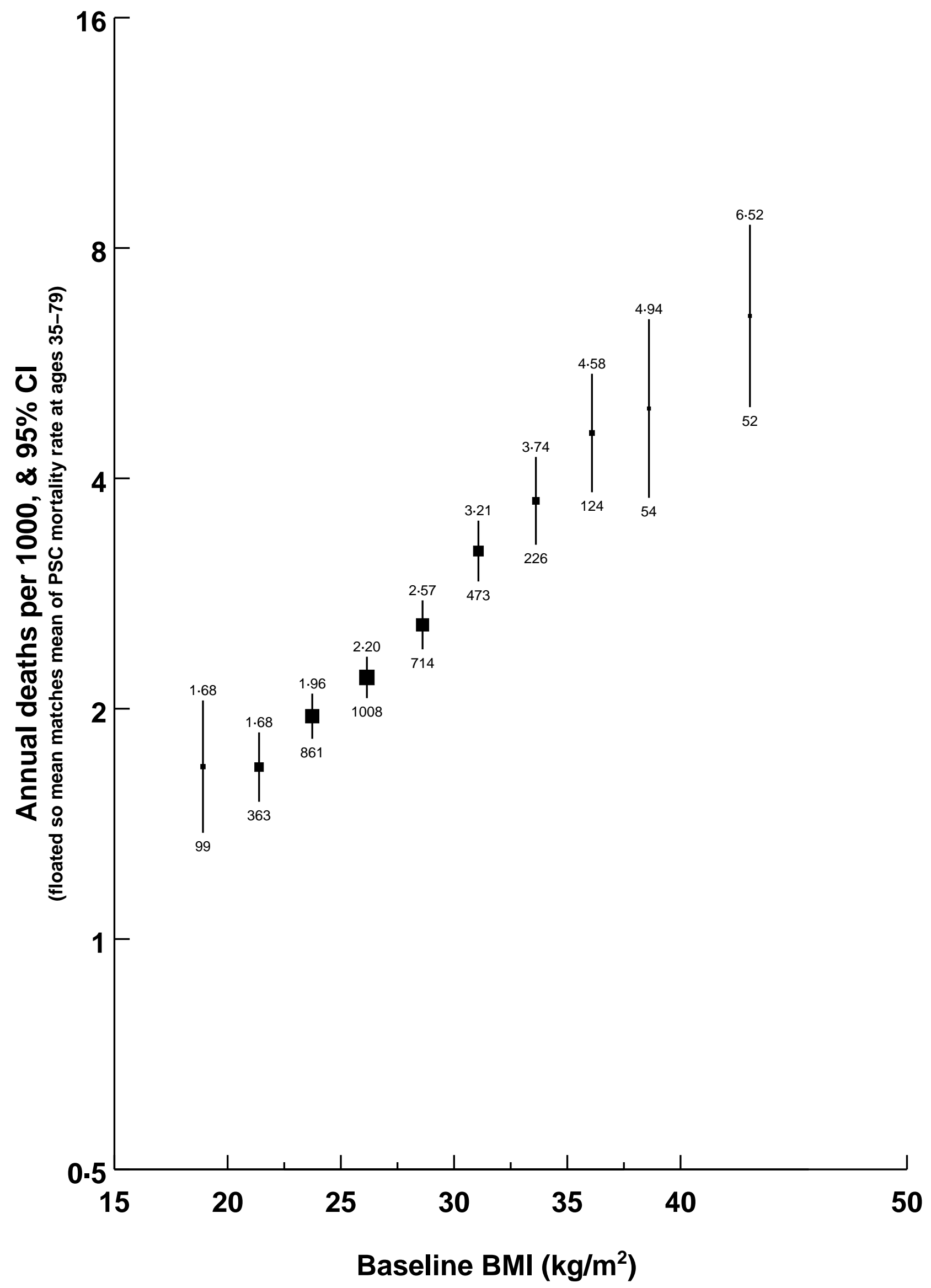


Webfigure 3: Stroke mortality vs. BMI in the range $15-50 \mathrm{~kg} / \mathrm{m}^{2}$, excluding (a) the first 5 years of follow-up,

(b) the first 10 years of follow-up, or (c) the first 5 years of follow-up and those who had ever smoked

Conventions as in figure 2, except (c) not adjusted for smoking.

(a) Excluding first 5 years of follow-up

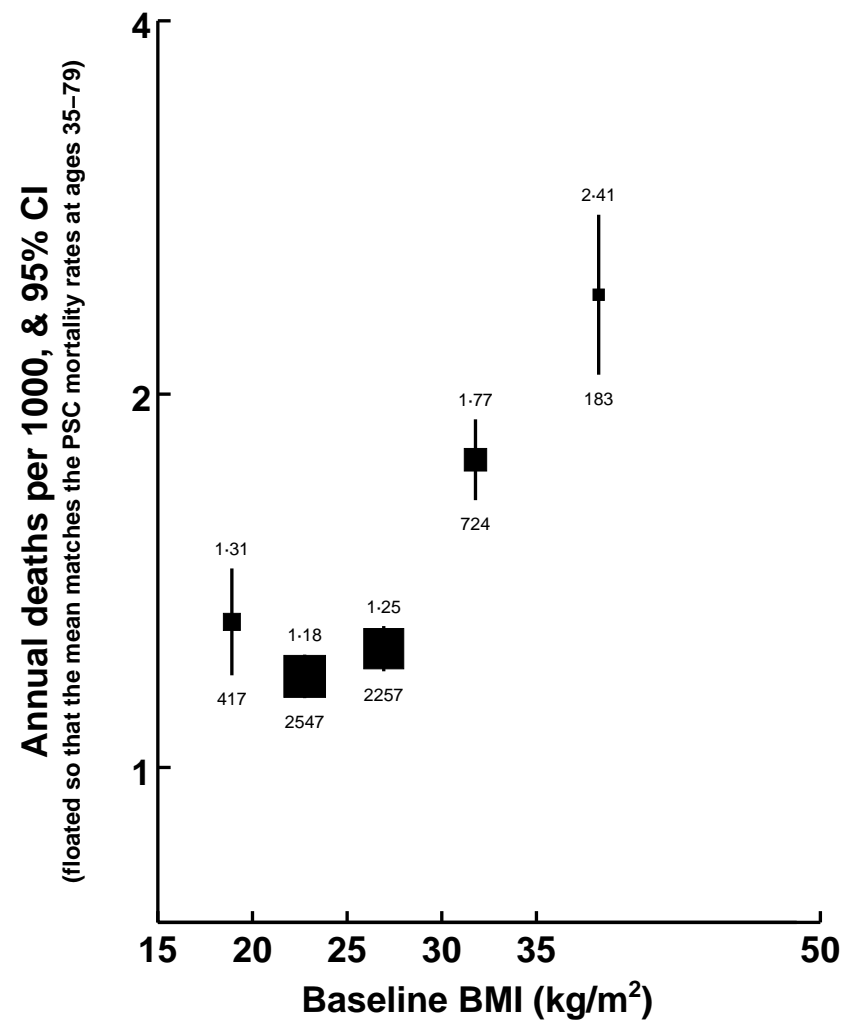

(b) Excluding first 10 years of follow-up

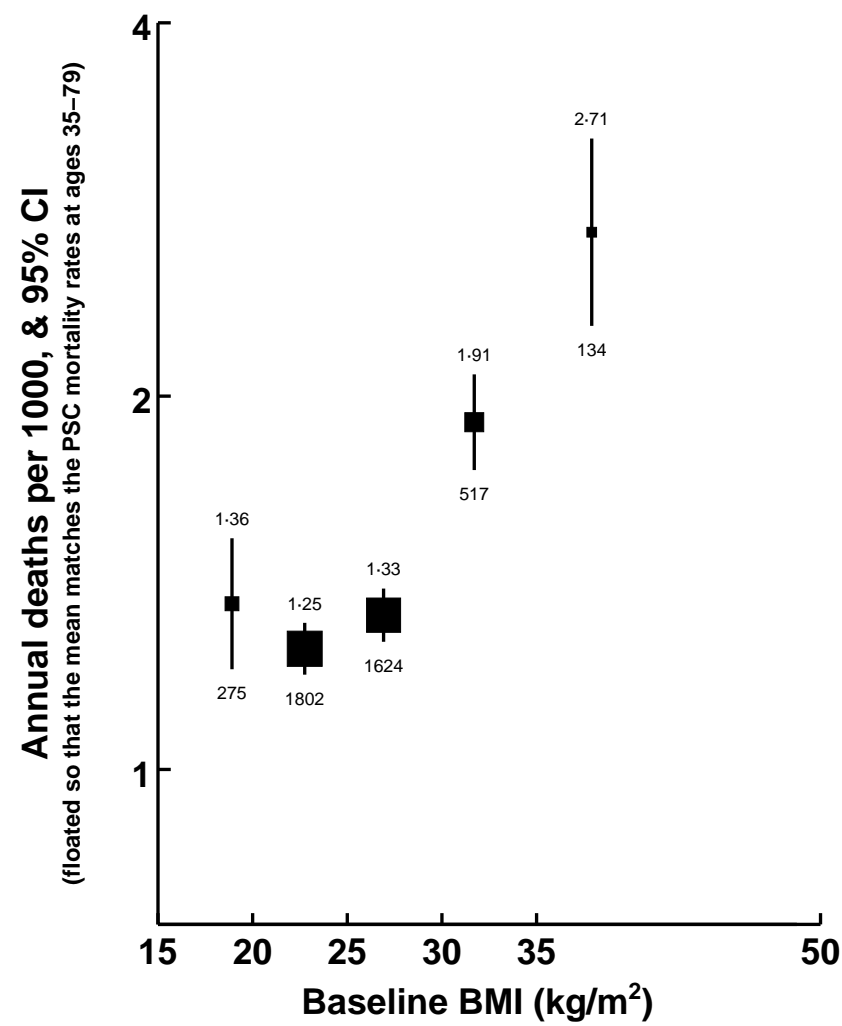

(c) Excluding first 5 years and ever smokers

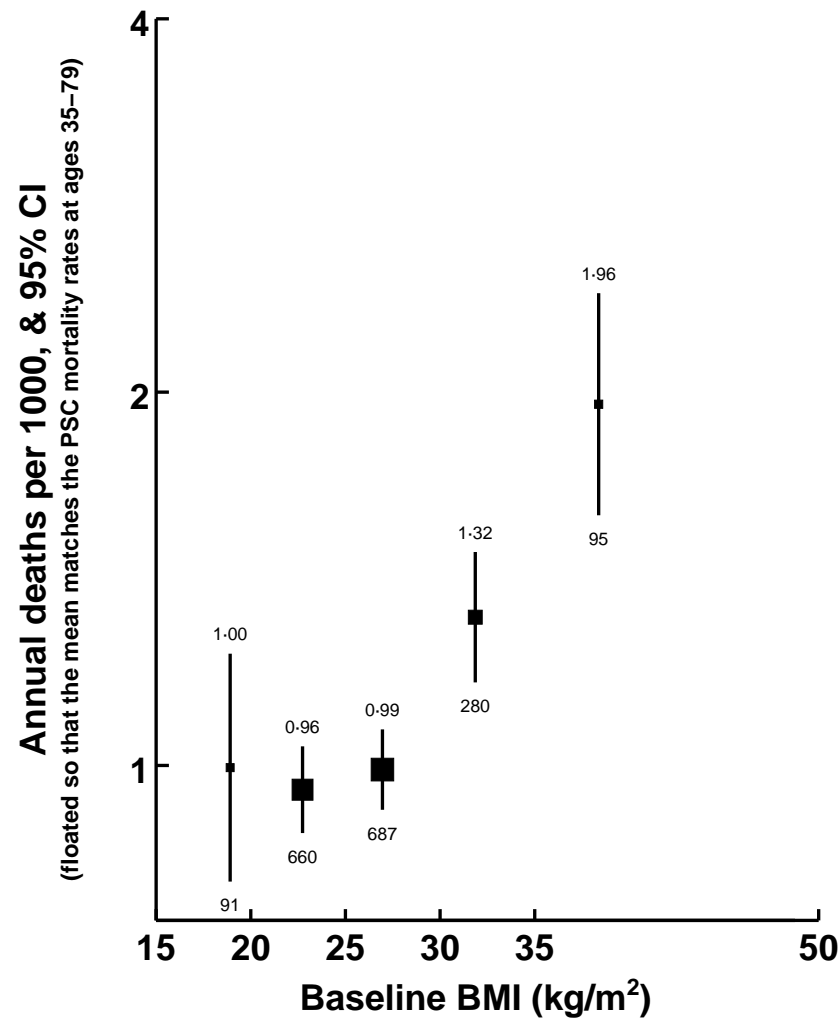


Webfigure 4: Stroke subtype mortality vs. BMI in the range $15-50 \mathrm{~kg} / \mathrm{m}^{2}$ (excluding the first 5 years of follow-up)

Conventions as in figure 2.

(a) Ischaemic

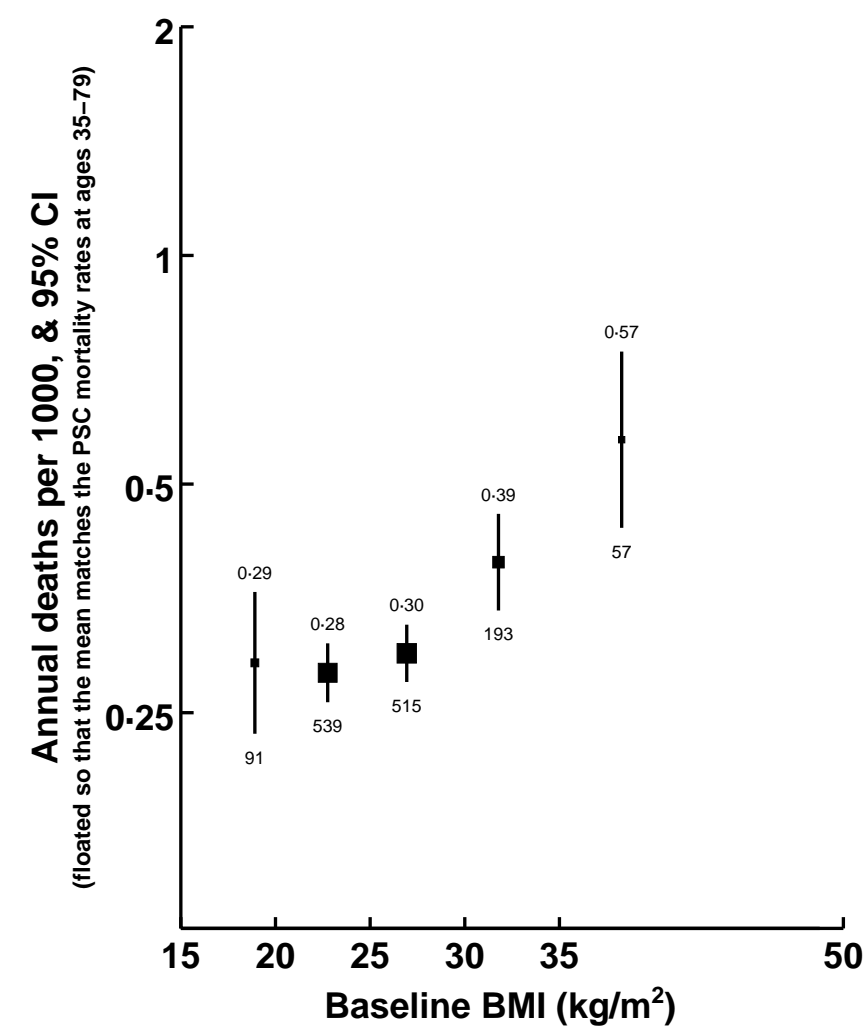

(b) Haemorrhagic

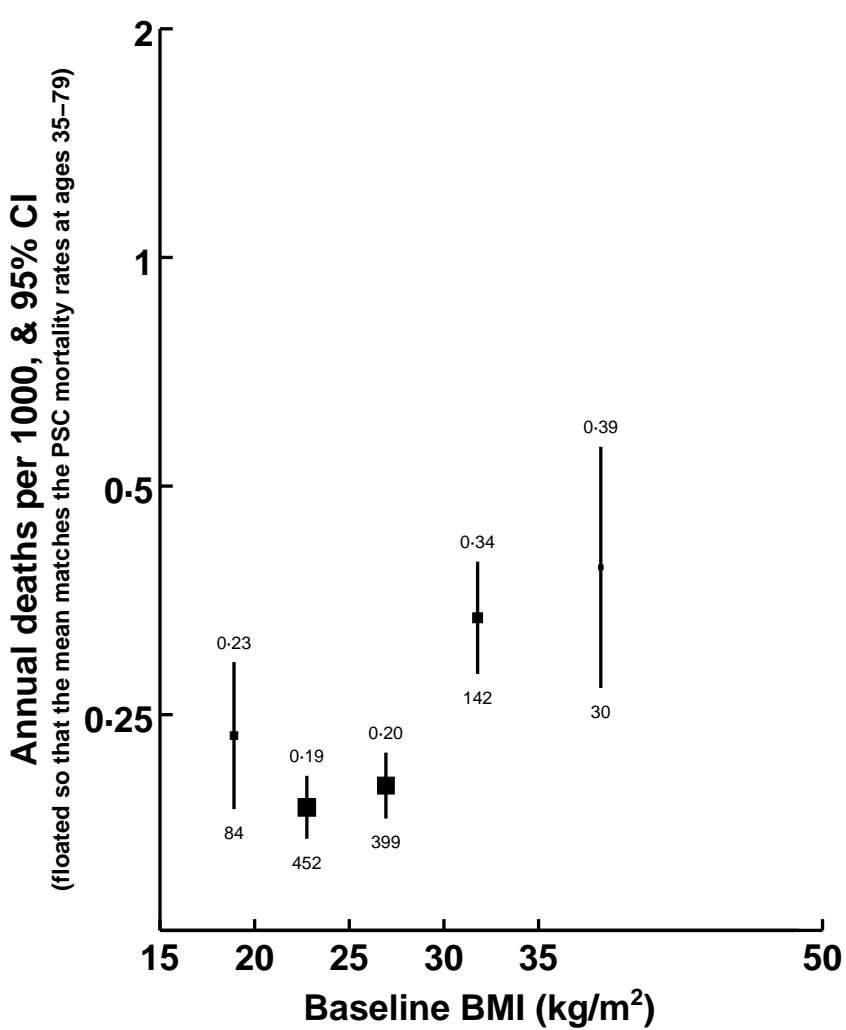

(c) Unclassified

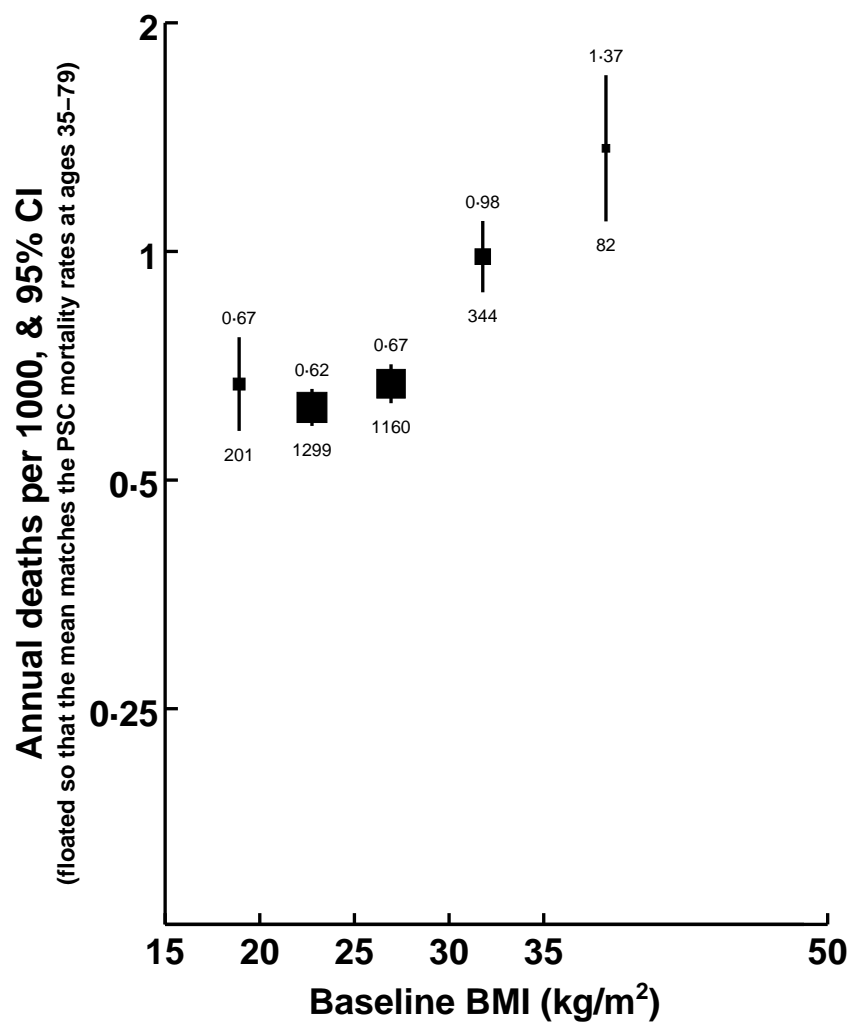


Webfigure 5: IHD and stroke mortality vs. BMI, by study:

hazard ratio per $5 \mathrm{~kg} / \mathrm{m}^{2}$ higher BMI in the range $25-50 \mathrm{~kg} / \mathrm{m}^{2}$

(excluding the first 5 years of follow-up)

Conventions as in figure 4, except: (i) hazard ratios adjusted for baseline age rather than age at risk (cf. Methods); and (ii) white squares represent inverse-variance-weighted averages for smaller studies. Dotted line indicates hazard ratio 1.39 (cf. IHD and stroke results in webtable 6). Study names as in webtable 1.

$\begin{array}{lr}\text { a) IHD mortality } & \begin{array}{r}\text { No. of } \\ \text { deaths }\end{array} \\ \text { Finnish Mobile Clinic } & 1724 \\ \text { Finrisk, Finland } & 1014 \\ \text { UK HDPP } & 703 \\ \text { Israeli IHD } & 571 \\ \text { Whitehall, UK } & 568 \\ \text { NHEFS, US } & 494 \\ \text { Framingham, US } & 481 \\ \text { Renfrew/Paisley, UK } & 438 \\ \text { Norwegian Counties } & 438 \\ \text { BUPA, UK } & 362 \\ \text { Oslo, Norway } & 339 \\ \text { Midspan, UK } & 318 \\ \text { Copenhagen, Denmark } & 231 \\ \text { BRHS, UK } & 227 \\ \text { Busselton, Australia } & 222 \\ \text { Tecumseh, US } & 215 \\ \text { Studies with <200 deaths } & 2438\end{array}$

ALL STUDIES

10783

Test for heterogeneity: $\chi_{16}{ }^{2}=22.5(p=0 \cdot 13)$

\section{b) Stroke mortality}

Finnish Mobile Clinic

Finrisk, Finland

Israeli IHD

NHEFS, US

Renfrew/Paisley, UK

Framingham, US

UK HDPP

Honolulu, US

Studies with $<100$ deaths

ALL STUDIES

Test for heterogeneity: $\chi_{8}^{2}=6 \cdot 1(p=0.64)$

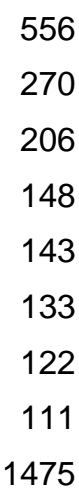

3164 556

270

206

148

143

133

122

111

1475

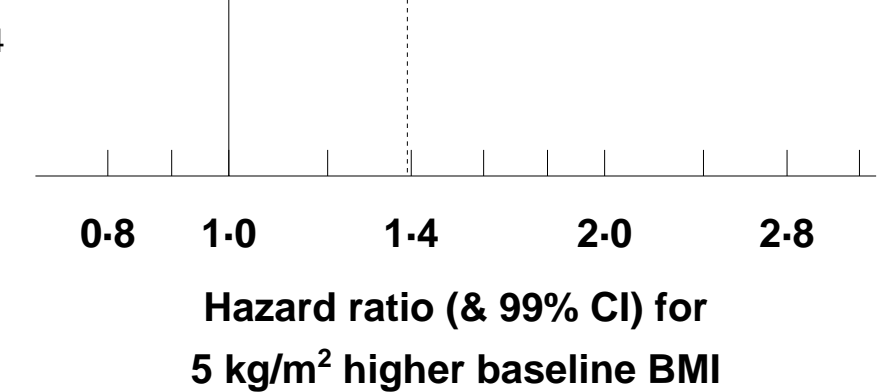


Webfigure 6: COPD mortality vs. BMI in the range $15-50 \mathrm{~kg} / \mathrm{m}^{2}$ (by follow-up period)

Conventions as in figure 2. Results for $35-50 \mathrm{~kg} / \mathrm{m}^{2}$ not plotted because of small numbers of deaths.

(a) 0-4 years (342 deaths)

(BMI 35-50 kg/m²: 4 deaths, not plotted)

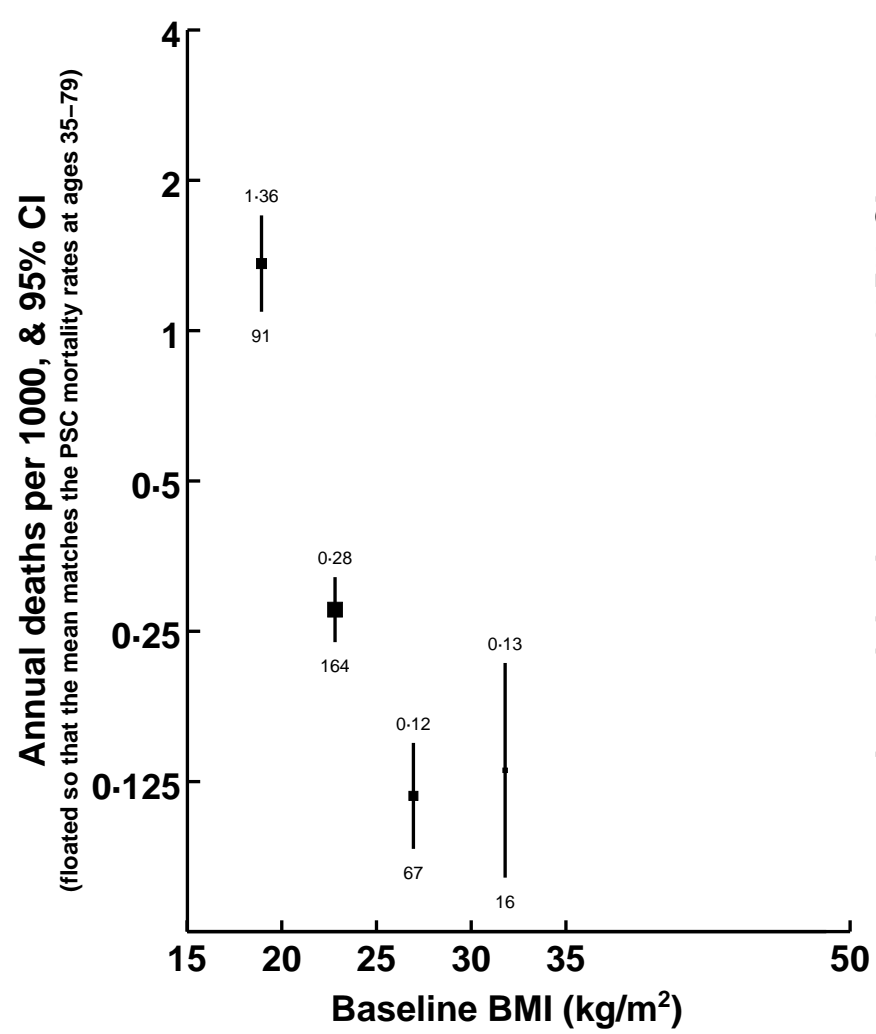

(c) 10-14 years (596 deaths)

(BMI 35-50 kg/m²: 7 deaths, not plotted)

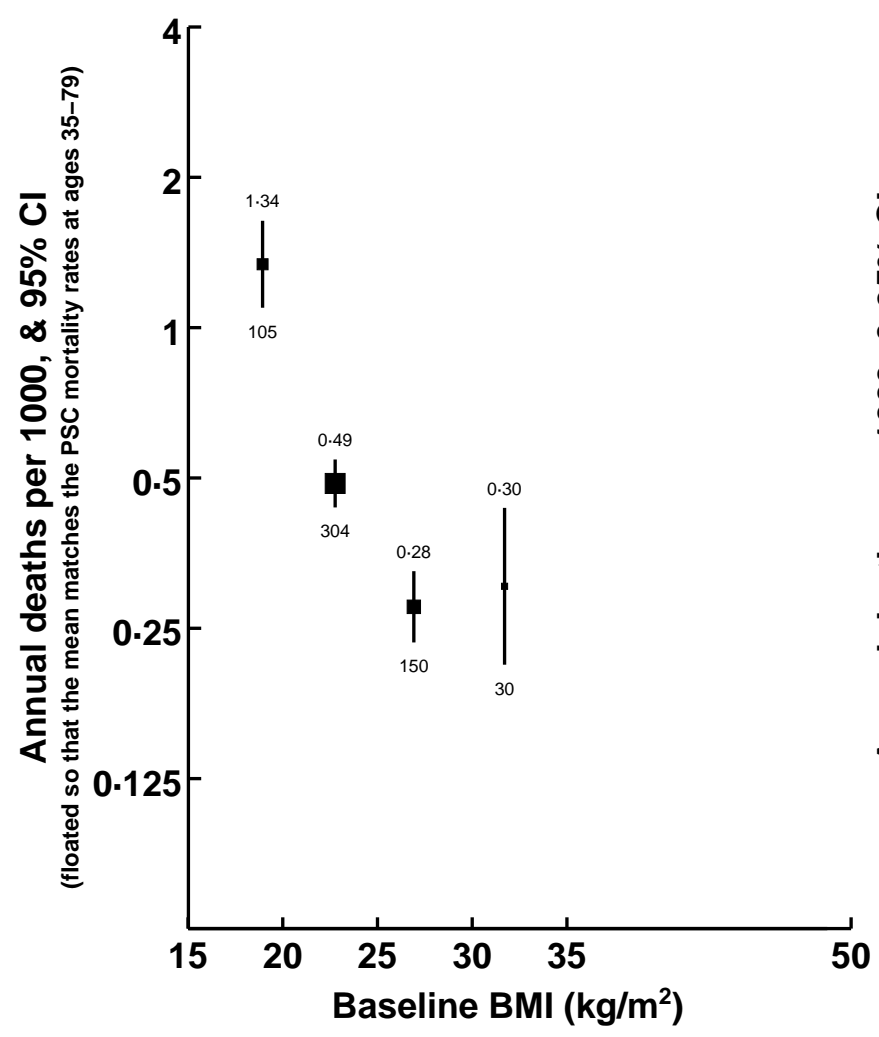

(b) 5-9 years (546 deaths)

(BMI 35-50 kg/m²: 6 deaths, not plotted)

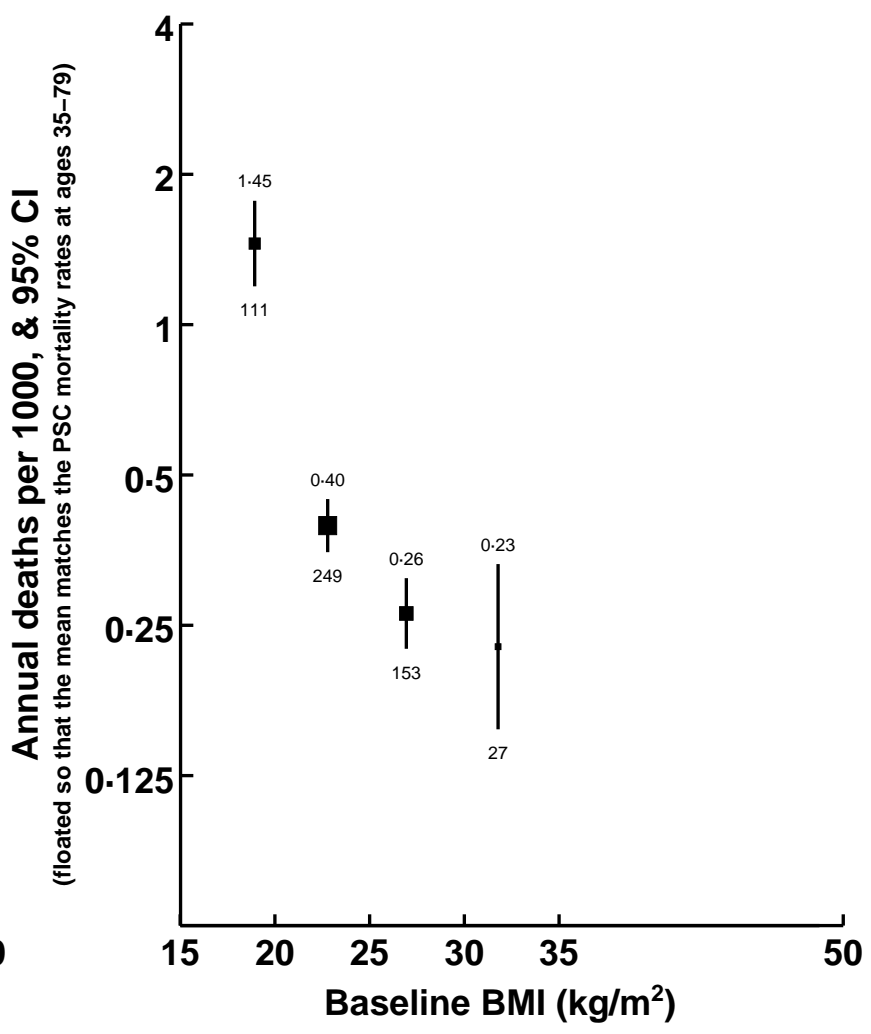

(d) 15+ years (812 deaths)

(BMI $35-50 \mathrm{~kg} / \mathrm{m}^{2}: 6$ deaths, not plotted)

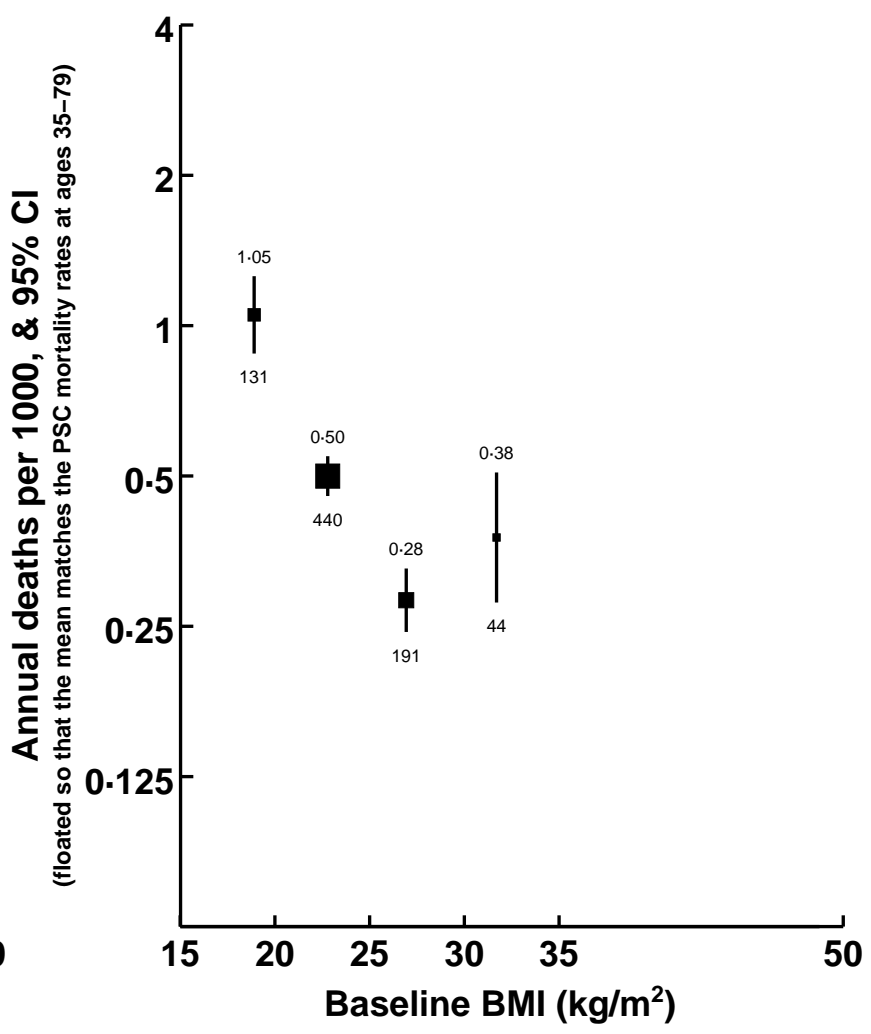


Webfigure 7: All-cause mortality vs. BMI by baseline smoking status (excluding the first 5 years of follow-up)

Conventions as in figure 6 . For $35-50 \mathrm{~kg} / \mathrm{m}^{2}$, only the upper or only the lower part of the CI is shown.

(a) Male (35-69 years)

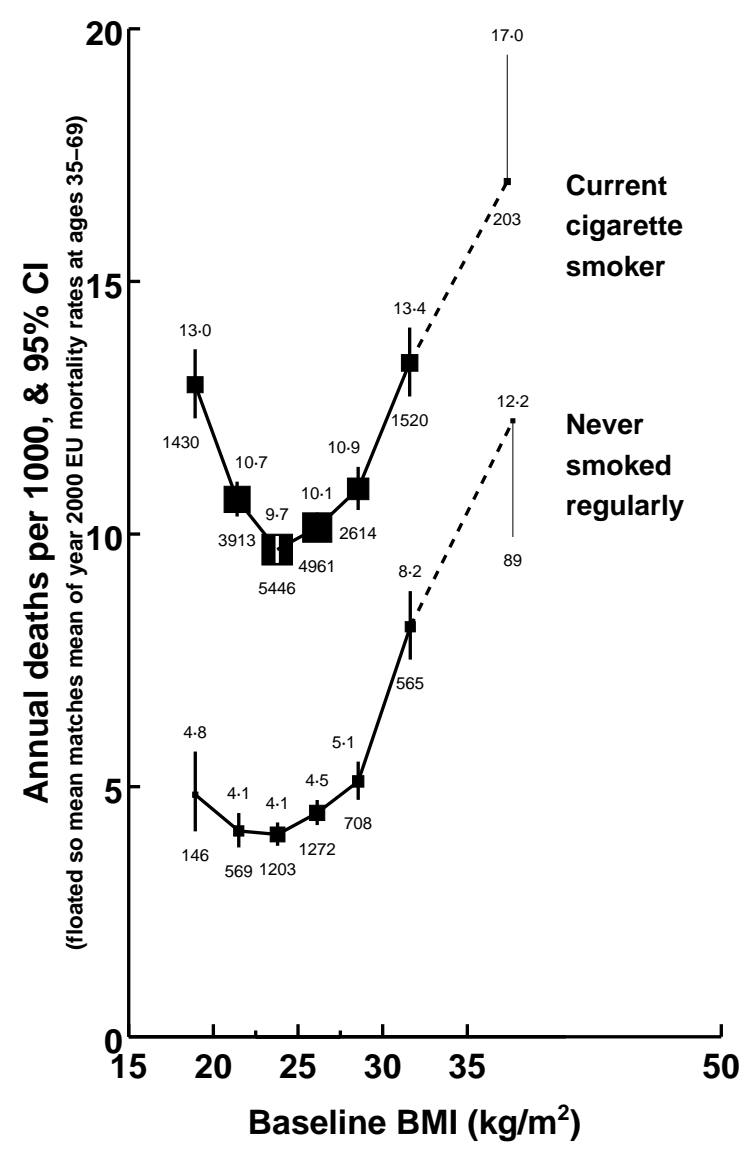

(c) Male (70-79 years)

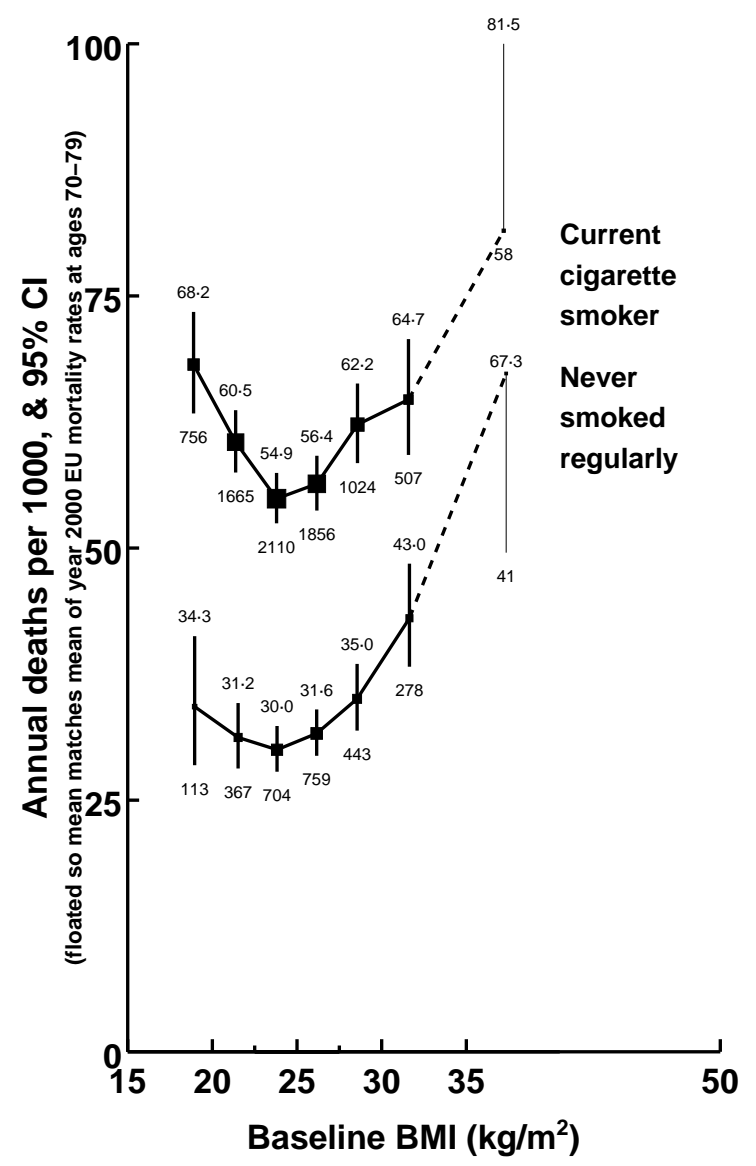

(b) Female (35-69 years)

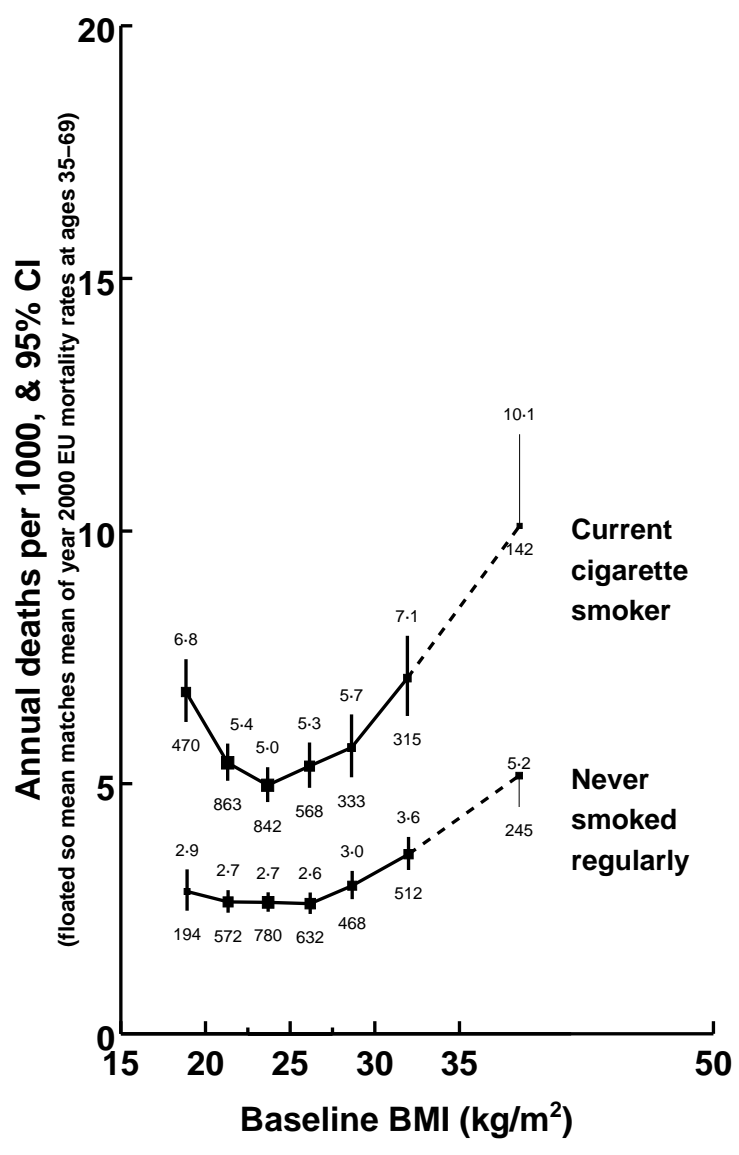

(d) Female (70-79 years)

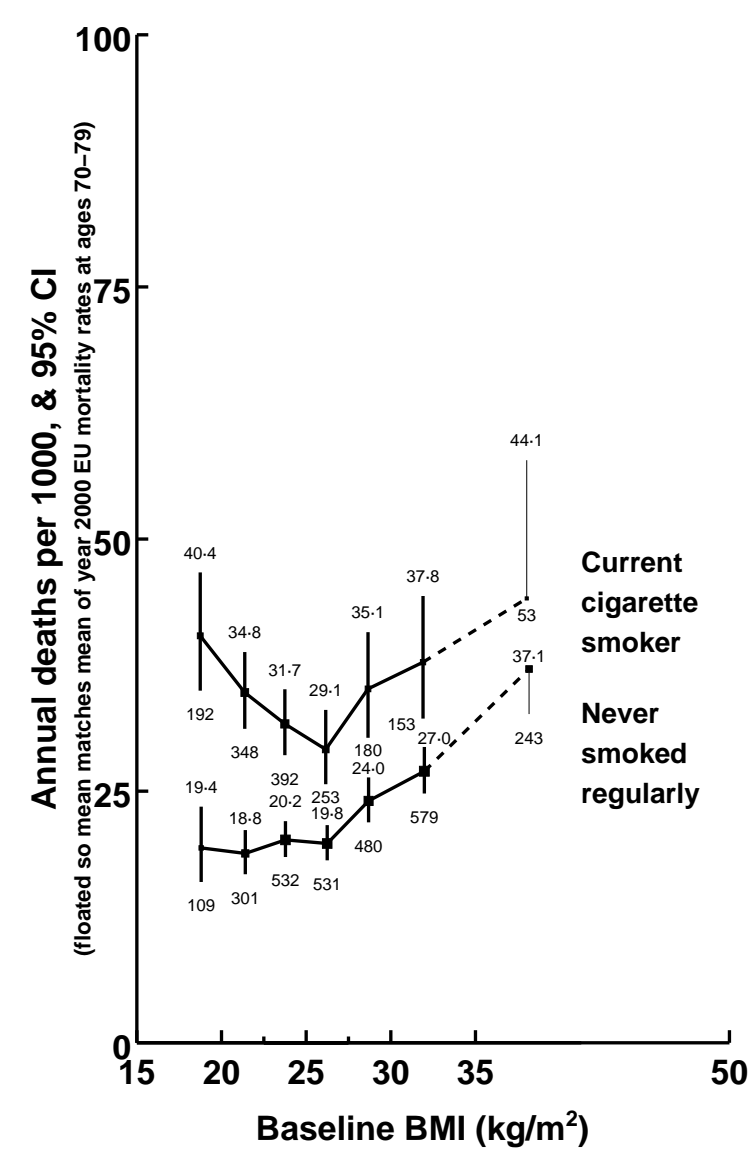


Page 8 of PSC BMI webappendix (Lancet 2009)

\section{Webfigure 8: Vascular mortality vs. BMI by baseline smoking status} (excluding the first 5 years of follow-up)

Conventions as in figure 6 . Results for $35-50 \mathrm{~kg} / \mathrm{m}^{2}$ not plotted because of small numbers of deaths.

(a) Male (35-69 years)

(BMI $35-50 \mathrm{~kg} / \mathrm{m}^{2}: 147$ deaths, not plotted)

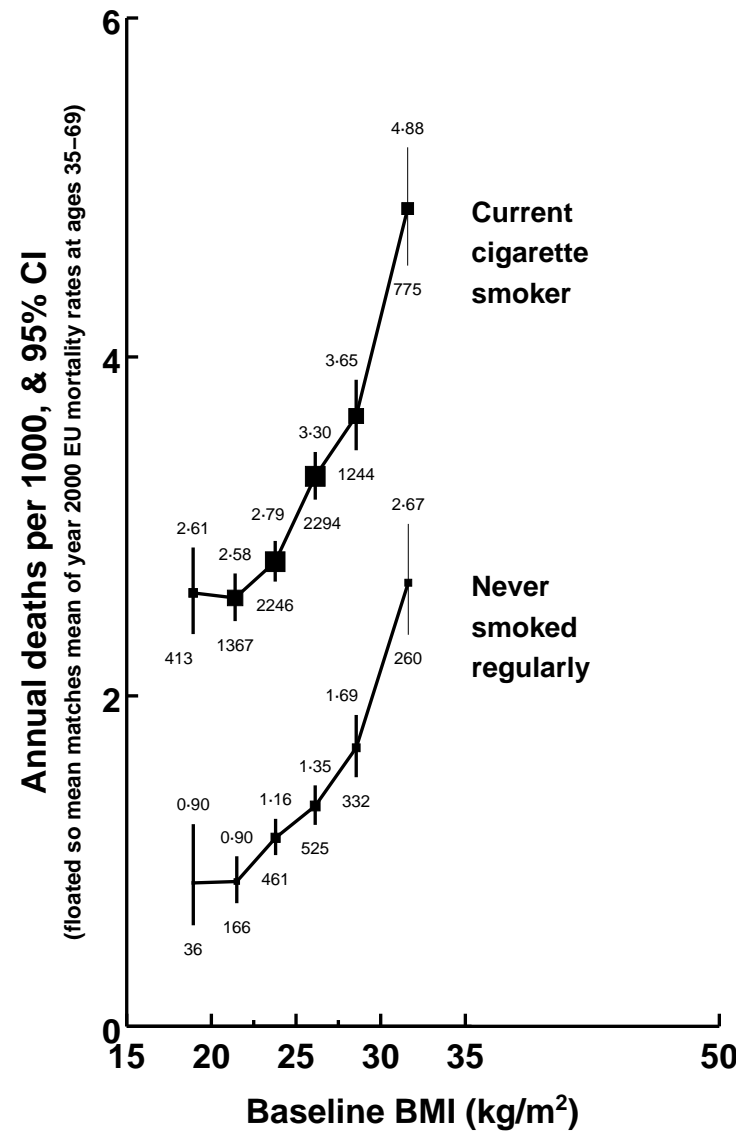

(c) Male (70-79 years)

(BMI $35-50 \mathrm{~kg} / \mathrm{m}^{2}: 46$ deaths, not plotted)

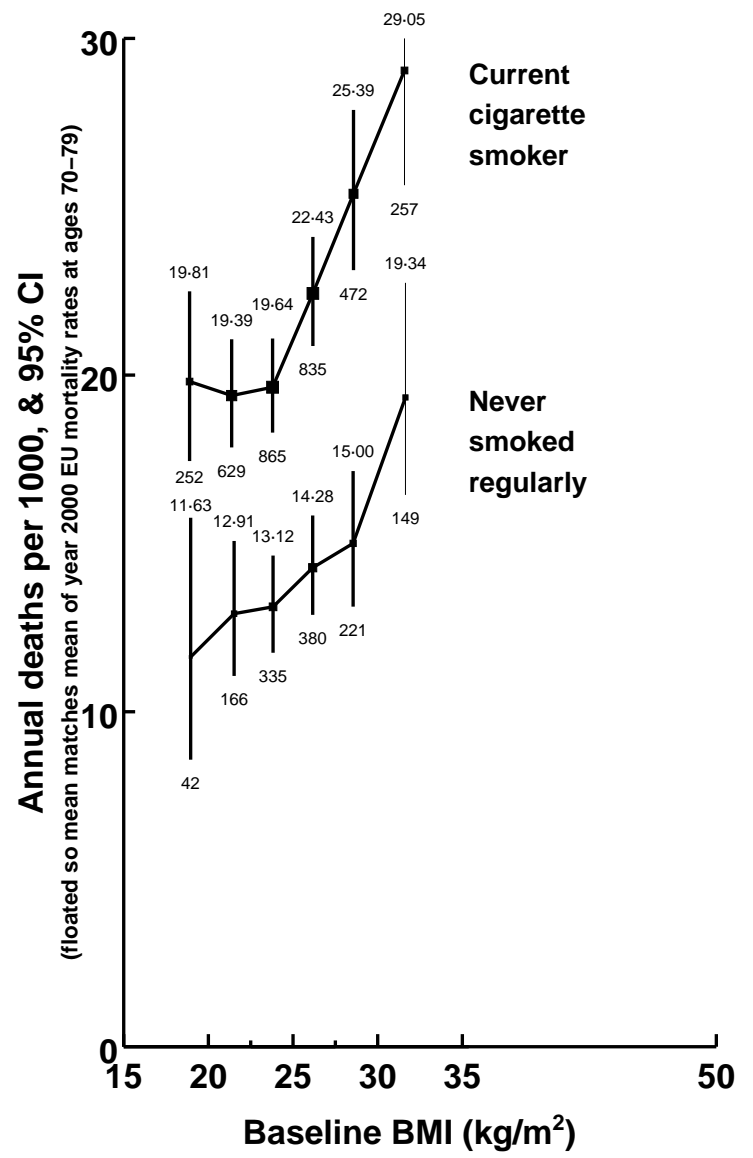

(b) Female (35-69 years)

(BMI $35-50 \mathrm{~kg} / \mathrm{m}^{2}: 178$ deaths, not plotted)

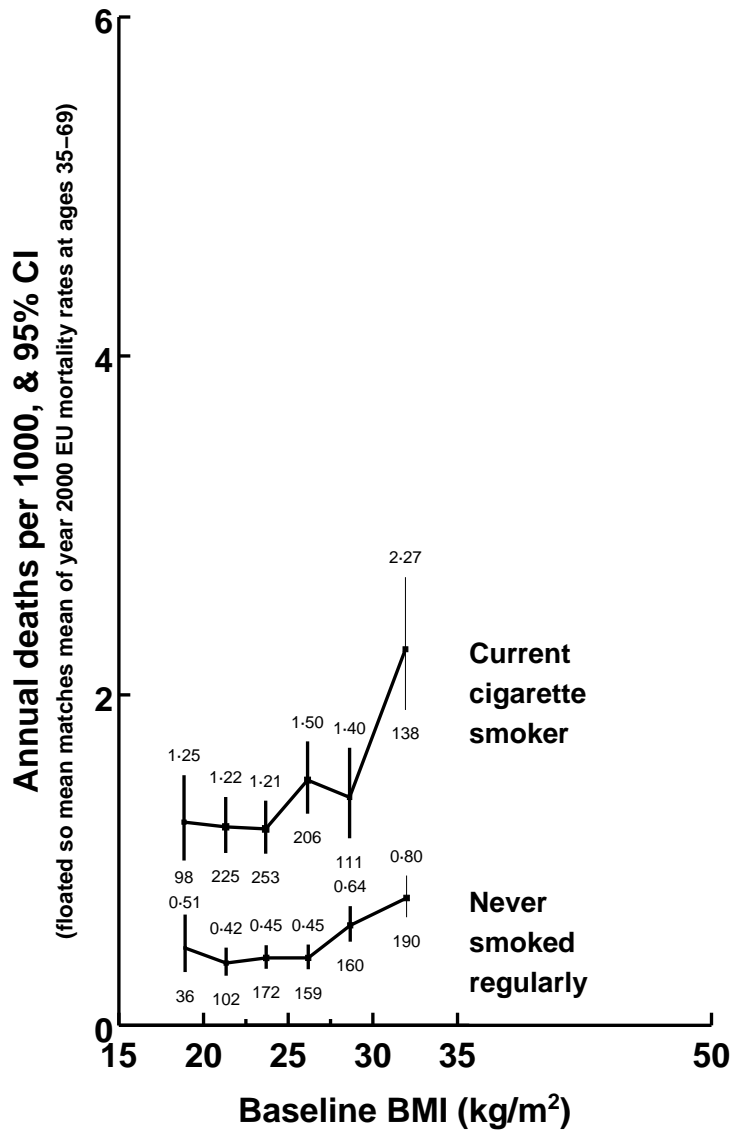

(d) Female (70-79 years)

(BMI $35-50 \mathrm{~kg} / \mathrm{m}^{2}: 174$ deaths, not plotted)

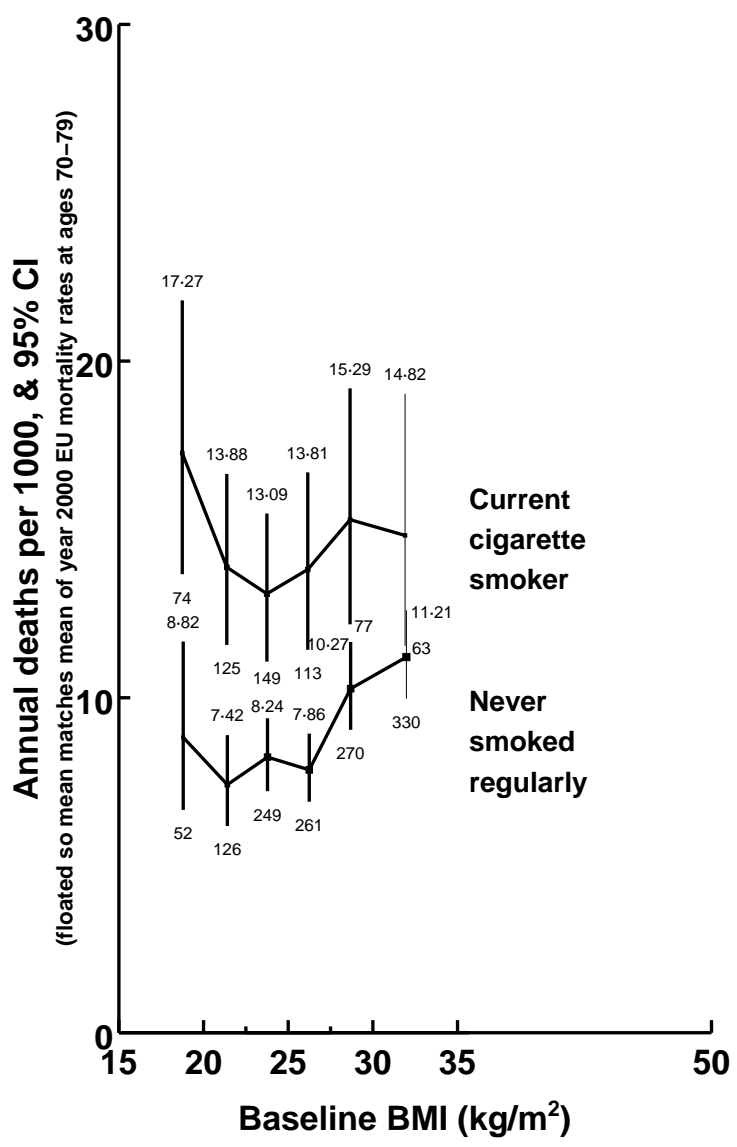


Webtable 1: Characteristics of 56 collaborating studies with data on BMI*

\begin{tabular}{|c|c|c|c|c|c|c|c|}
\hline \multirow[b]{2}{*}{$\begin{array}{l}\text { Study, by region and median } \\
\text { year of assessment }\end{array}$} & \multicolumn{4}{|c|}{ Baseline } & \multicolumn{3}{|c|}{ Excluding 1st 5 years of follow-up } \\
\hline & $\begin{array}{l}\text { Median year of } \\
\text { assessment }\end{array}$ & $\begin{array}{c}\text { Number of } \\
\text { participants }\end{array}$ & $\begin{array}{c}\% \\
\text { Female }\end{array}$ & $\begin{array}{l}\text { BMI, mean } \\
(\mathrm{SD}), \mathrm{kg} / \mathrm{m}^{2}\end{array}$ & $\begin{array}{l}\text { Number of } \\
\text { deaths }\end{array}$ & $\begin{array}{l}\text { Mean (SD) } \\
\text { age at death }\end{array}$ & $\begin{array}{c}\text { Median year } \\
\text { of death }\end{array}$ \\
\hline \multicolumn{8}{|l|}{ Europe and Israel (32 studies) } \\
\hline Seven Countries, Croatia & 1958 & 1305 & 0 & $23.0(3.2)$ & 645 & 68 (7) & 1976 \\
\hline Seven Countries, Finland & 1959 & 1487 & 0 & $23.7(3.2)$ & 712 & $67(7)$ & 1976 \\
\hline Seven Countries, Greece & 1960 & 1155 & 0 & $23.1(3.3)$ & 371 & $69(7)$ & 1979 \\
\hline Seven Countries, Italy & 1960 & 2310 & 0 & $25.6(3.7)$ & 897 & $67(7)$ & 1977 \\
\hline Seven Countries, Netherlands & 1960 & 829 & 0 & $24.0(2.7)$ & 360 & $68(7)$ & 1978 \\
\hline Israeli IHD & 1963 & 9849 & 0 & $25.6(3.3)$ & 3085 & $68(8)$ & 1979 \\
\hline Seven Countries, Serbia & 1963 & 964 & 0 & $23.7(3.5)$ & 476 & $68(7)$ & 1980 \\
\hline Whitehall, UK & 1968 & 18863 & 0 & $24.7(3.0)$ & 2737 & $66(7)$ & 1979 \\
\hline Gothenburg Women, Sweden & 1969 & 1406 & 100 & $24.0(3.7)$ & 259 & $68(8)$ & 1988 \\
\hline Finnish Mobile Clinic & 1970 & 46815 & 47 & $24.5(4.0)$ & 7903 & $68(12)$ & $\dagger$ \\
\hline Paris PS, France & 1970 & 7517 & 0 & $25.9(3.3)$ & 1938 & $64(6)$ & 1986 \\
\hline Midspan, UK & 1971 & 6602 & 14 & $25.0(3.1)$ & 1714 & $66(8)$ & 1987 \\
\hline UK HDPP & 1972 & 13398 & 0 & $25.3(3.2)$ & 3410 & $68(7)$ & 1990 \\
\hline Oslo, Norway & 1973 & 15692 & 0 & 24.7 (2.9) & 1738 & $58(5)$ & 1986 \\
\hline Northwick Park HS, UK & 1975 & 3334 & 31 & 24.9 (3.3) & 446 & $68(9)$ & 1988 \\
\hline Renfrew/Paisley, UK & 1975 & 11205 & 55 & $25.6(3.8)$ & 2320 & $68(6)$ & 1986 \\
\hline Norwegian Counties & 1976 & 46805 & 50 & $24.9(3.6)$ & 2791 & $55(5)$ & $\dagger$ \\
\hline CB Project, Netherlands & 1977 & 48725 & 52 & $24.6(3.4)$ & 1083 & $52(6)$ & 1988 \\
\hline Copenhagen, Denmark & 1977 & 13749 & 55 & $25.2(4.2)$ & 3640 & $71(9)$ & 1989 \\
\hline Finrisk, Finland & 1977 & 37573 & 52 & $26.1(4.2)$ & 3973 & $63(9)$ & 1988 \\
\hline BUPA, UK & 1978 & 21187 & 0 & $25.1(2.8)$ & 1862 & $65(8)$ & 1990 \\
\hline BRHS, UK & 1979 & 7401 & 0 & $25.5(3.2)$ & 1029 & $63(6)$ & 1990 \\
\hline Tromso, Norway & 1979 & 14361 & 48 & $23.7(3.2)$ & 275 & $53(7)$ & 1988 \\
\hline OG-Rome, Italy & 1980 & 3168 & 0 & $26.5(3.2)$ & 225 & $65(5)$ & 1987 \\
\hline Speedwell, UK & 1980 & 1870 & 0 & $25.5(3.2)$ & 256 & $66(5)$ & 1991 \\
\hline Caerphilly, UK & 1981 & 1891 & 0 & $26.0(3.5)$ & 240 & $63(5)$ & 1991 \\
\hline IPC-Paris, France & 1982 & 180039 & 44 & $23.8(3.4)$ & 3860 & $60(12)$ & 1991 \\
\hline BIRNH, Belgium & 1983 & 10559 & 48 & $25.9(4.1)$ & 575 & $70(9)$ & 1990 \\
\hline Glostrup, Denmark & 1983 & 9140 & 50 & $24.8(4.0)$ & 529 & $68(12)$ & 1990 \\
\hline PROCAM, Germany & 1983 & 13580 & 24 & $26.0(3.4)$ & 176 & $59(7)$ & 1990 \\
\hline Scottish HHS & 1986 & 12514 & 51 & $25.7(4.1)$ & 523 & $60(6)$ & 1993 \\
\hline FLEMENGHO (Leuven), Belgium & 1987 & 1212 & 51 & $25.9(4.1)$ & 50 & $73(10)$ & 1993 \\
\hline Subtotals \& averages $\ddagger$ & $1978(1972 \S)$ & 566505 & 37 & 24.7 (3.6) & 50098 & $65(10)$ & 1987 \\
\hline \multicolumn{8}{|c|}{ United States and Australia (17 studies) } \\
\hline Framingham, US & 1950 & 5072 & 56 & $25.5(4.1)$ & 2920 & $72(10)$ & 1977 \\
\hline Tecumseh, US & 1960 & 4288 & 52 & $25.8(4.6)$ & 1241 & $70(11)$ & 1977 \\
\hline Charleston, US & 1961 & 2066 & 56 & $25.4(4.8)$ & 1117 & $71(10)$ & 1980 \\
\hline Honolulu, US & 1967 & 7582 & 0 & $23.8(3.1)$ & 2866 & $74(8)$ & 1985 \\
\hline Puerto Rico HHP, US (admin) & 1967 & 9167 & 0 & $25.1(4.1)$ & 1156 & $66(7)$ & 1976 \\
\hline Busselton, Australia & 1969 & 6542 & 52 & $24.5(3.7)$ & 1428 & $74(11)$ & 1986 \\
\hline NHEFS, US & 1973 & 12979 & 60 & 25.5 (4.9) & 3131 & $74(11)$ & 1985 \\
\hline Rancho Bernardo, US & 1973 & 5333 & 56 & 24.4 (3.5) & 1409 & $78(9)$ & 1986 \\
\hline Lipid Research Clinic, US & 1974 & 8165 & 46 & $26.0(4.2)$ & 995 & $69(12)$ & 1985 \\
\hline Physicians' HS, US & 1982 & 22058 & 0 & $24.9(3.0)$ & 1435 & $71(10)$ & 1991 \\
\hline Perth, Australia & 1983 & 9272 & 49 & 25.2 (3.9) & 552 & $69(11)$ & 1993 \\
\hline Minnesota HHP, US & 1984 & 15164 & 55 & $26.1(4.7)$ & 213 & $69(11)$ & 1989 \\
\hline Minnesota HS, US & 1985 & 8494 & 54 & $26.4(4.8)$ & 116 & $67(11)$ & 1989 \\
\hline Health Professionals, US & 1986 & 46717 & 0 & $25.5(3.1)$ & 648 & $67(9)$ & 1992 \\
\hline ARIC, US & 1988 & 14774 & 57 & $27.5(5.2)$ & 474 & $64(5)$ & 1995 \\
\hline Nurses' HS, US** & 1988 & 78069 & 100 & $25.6(4.8)$ & 417 & $64(6)$ & 1993 \\
\hline CHS, US & 1989 & 4493 & 60 & $26.4(4.6)$ & 25 & $77(6)$ & 1994 \\
\hline Subtotals \& averages $\ddagger$ & $1986(1968 \S)$ & 260235 & 50 & $25.6(4.3)$ & 20143 & $72(10)$ & 1985 \\
\hline \multicolumn{8}{|l|}{ Japan† (8 studies) } \\
\hline Seven Countries, Japan & 1958 & 954 & 0 & $22.0(2.4)$ & 399 & $68(7)$ & 1975 \\
\hline Japan Railway: EJR & 1976 & 50039 & 0 & $22.7(2.7)$ & 857 & $55(4)$ & 1982 \\
\hline Noichi & 1976 & 2180 & 63 & $22.9(3.2)$ & 290 & $72(9)$ & 1988 \\
\hline Ikawa & 1977 & 2098 & 56 & $23.6(3.2)$ & 266 & $71(9)$ & 1988 \\
\hline Shibata & 1977 & 2246 & 58 & $22.4(3.0)$ & 388 & $75(10)$ & 1987 \\
\hline Kyowa & 1983 & 4099 & 57 & $23.5(3.3)$ & 184 & $68(8)$ & 1991 \\
\hline Saitama & 1987 & 3406 & 63 & $22.4(2.9)$ & 109 & $72(10)$ & 1993 \\
\hline Ohasama & 1990 & 2814 & 62 & $23.6(3.1)$ & 15 & $74(12)$ & 1995 \\
\hline Subtotals \& averages $\ddagger$ & 1977 (1975§) & 67836 & 15 & $22.8(2.9)$ & 2508 & $66(11)$ & 1984 \\
\hline TOTALS \& AVERAGES $\ddagger$ & $1979(1972 \S)$ & 894576 & 39 & $24.8(3.8)$ & 72749 & $67(11) \dagger \dagger$ & 1986 \\
\hline
\end{tabular}

* References to all 61 PSC studies are in Lancet 2002;360:1903-13 (Appendix A). Excluded from these BMI analyses are 3 studies without BMI data (MRFIT,

Evans County and Shanghai), 1 study with BMI data in broad categories only (Värmland), and 1 study without IHD mortality data (Seven Cities China).

† Data unavailable. Median year of death during all follow-up: 1985 (Finnish Mobile Clinic), 1987 (Norwegian Counties).

¥ Where available; weighted for number of individuals

$\S$ Median year of assessment of those who died after 5 or more years of follow-up

** Nurses who had a cholesterol measurement in about 1988

†† For the 66552 deaths of known cause, the mean (SD) age at death was 67 (10) years 
Webtable 2: Mortality endpoints and their ICD-9* codes

\begin{tabular}{|c|c|c|}
\hline Endpoint name & ICD-9 codes & Notes \\
\hline ALL VASCULAR DISEASE & $390-459,798$ & Includes sudden death and hypertensive renal disease \\
\hline Ischaemic heart disease (IHD) & $410-414$ & \\
\hline Stroke & $430-438$ & \\
\hline Ischaemic stroke & $433-434$ & \\
\hline Haemorrhagic stroke & $431-432$ & Mainly intracerebral haemorrhage (and excludes subarachnoid haem.) \\
\hline Subarachnoid haemorrhage & 430 & \\
\hline Unclassified stroke & $435-438$ & Includes (miscertified) transient cerebral ischaemia \\
\hline Other vascular disease & Remainder of vascular & Some such deaths could not be further subdivided in the PSC \\
\hline Aortic aneurysm & 441 & \\
\hline Pulmonary embolism & 415 & Includes acute cor pulmonale, but not deep vein thrombosis \\
\hline Heart failure & 428 & \\
\hline Hypertensive disease & $401-405$ & Includes essential hypertension and hypertensive renal disease \\
\hline Atherosclerosis \& other arterial disease & $440,442-448$ & Includes peripheral arterial disease, \& diseases of arterioles/capillaries \\
\hline Inflammatory heart disease & $420-424$ & Includes pericarditis, myocarditis, endocarditis \\
\hline Rheumatic heart disease & $390-398$ & \\
\hline Sudden death & 798 & \\
\hline Other heart disease (not IHD) & $416-417,425-427,429$ & Includes pulmonary heart disease, cardiomyopathy, dysrhythmia \\
\hline Other circulatory disease & $451-459$ & Includes venous disease \\
\hline Diabetes (any type) & 250 & In some countries, rarely recorded as the underlying cause \\
\hline Kidney disease (non-neoplastic) & $580-589$ & Excludes hypertensive renal disease \\
\hline Liver disease (non-neoplastic) & $070,570-573$ & \\
\hline Cirrhosis & 571 & \\
\hline ALL NEOPLASTIC DISEASE & $140-208$ & Excludes benign neoplasms \\
\hline Liver cancer & 155 & Includes intrahepatic bile ducts; excludes known secondary cancers \\
\hline Kidney cancer & 189 & \\
\hline Female breast cancer, age $<60$ & 174 & Deaths at ages $35-59$ only \\
\hline Female breast cancer, age $\geq 60$ & 174 & Deaths at ages $60-89$ only \\
\hline Ovarian cancer & 183 & \\
\hline Endometrial cancer & 182 & Includes all cancers of body of the uterus \\
\hline Prostate cancer & 185 & \\
\hline Pancreatic cancer & 157 & \\
\hline Gallbladder cancer & 156 & Includes extrahepatic bile ducts \\
\hline Stomach cancer & 151 & \\
\hline Colorectal cancer & $153-154$ & \\
\hline Upper aerodigestive cancer & $140-150,161$ & Mouth, pharynx, larynx, oesophagus \\
\hline Oesophageal cancer & 150 & \\
\hline Lung cancer & 162 & Includes trachea and bronchus, but not pleura \\
\hline Haematological cancer & 200-208 & \\
\hline Leukaemia & 204-208 & \\
\hline Non-Hodgkin's lymphoma & 202 & \\
\hline Multiple myeloma & 203 & \\
\hline Unspecified cancer & $195-199$ & Unspecified to the PSC \\
\hline Other specified cancer (in webtable $7 \dagger$ ) & Remainder of neoplastic & Excludes benign neoplasms \\
\hline ALL RESPIRATORY DISEASE & $010-012,460-519$ & Includes influenza \\
\hline Chronic obstructive pulmonary disease & $490-496$ & Includes asthma \\
\hline Pneumonia & $480-486$ & \\
\hline Respiratory tuberculosis & 010-012 & \\
\hline Other respiratory disease & $\begin{array}{l}\text { Remainder of } \\
\text { respiratory }\end{array}$ & Includes influenza \\
\hline Other specified disease & Remainder of $001-779$ & \\
\hline External cause & (E)800-999 & Mostly injury \\
\hline Unknown cause (in webtable $7 \dagger$ ) & $780-797,799$ & Unknown to the PSC \\
\hline ALL CAUSES & 001-999 & \\
\hline
\end{tabular}

* Not all studies provided ICD-9 codes. When other ICD versions or the study's own codes were provided, these were grouped to approximate as closely as possible the endpoints shown here.

$\dagger$ In table 2 and webtable 9, other specified cancer was defined as all specified cancers other than lung and upper aerodigestive cancer. In table 2, unknown cause was defined as ICD-9 codes 195-199, 780-797 and 799. 
Webtable 3: Change in BMI from baseline to a repeat measurement, by age and sex

\begin{tabular}{|c|c|c|c|c|c|c|c|c|c|c|c|c|c|}
\hline & \multicolumn{6}{|c|}{$\begin{array}{l}\text { No. of people at baseline, and their } \\
\text { mean age, height and BMI:* }\end{array}$} & \multicolumn{7}{|c|}{$\begin{array}{l}\text { No. of people resurveyed, and their } \\
\text { change in BMI from baseline to first repeat measurement at: }\end{array}$} \\
\hline & \multirow{2}{*}{$\begin{array}{l}\text { Baseline } \\
\text { age (years) }\end{array}$} & \multirow[b]{2}{*}{ No. } & \multirow{2}{*}{$\begin{array}{l}\text { Mean } \\
\text { age }\end{array}$} & \multirow{2}{*}{$\begin{array}{c}\text { Mean } \\
\text { year of } \\
\text { birth } \dagger\end{array}$} & \multirow{2}{*}{$\begin{array}{c}\text { Mean } \\
\text { height } \\
(\mathrm{cm})\end{array}$} & \multirow{2}{*}{$\begin{array}{c}\text { Mean } \\
\text { BMI } \\
\left(\mathrm{kg} / \mathrm{m}^{2}\right)\end{array}$} & \multicolumn{2}{|c|}{ 0-4 (mean 2.6) years } & \multicolumn{3}{|c|}{ 5-9 (mean 6.0) years } & \multicolumn{2}{|c|}{$\geq 10($ mean 13.1$)$ years } \\
\hline & & & & & & & No. & Change (SE) & No. & Change (SE) & $\mathbf{r}(\mathrm{SE})+$ & No. & Change (SE) \\
\hline \multirow[t]{8}{*}{ Male } & $15-19$ & 3510 & 17.2 & 1956 & 1.75 & 19.95 & 82 & $1.15(0.17)$ & 70 & $1.86(0.16)$ & $0.88(0.028)$ & 96 & $3.72(0.24)$ \\
\hline & $20-29$ & 27724 & 26.0 & 1953 & 1.76 & 23.29 & 1317 & $0.49(0.04)$ & 2222 & $1.01(0.04)$ & $0.87(0.005)$ & 733 & $2.41(0.10)$ \\
\hline & $30-39$ & 88766 & 35.3 & 1944 & 1.75 & 24.60 & 3517 & $0.30(0.02)$ & 5813 & $0.78(0.02)$ & $0.89(0.003)$ & 1058 & $1.42(0.07)$ \\
\hline & $40-49$ & 205599 & 44.4 & 1932 & 1.74 & 25.28 & 9290 & $0.11(0.01)$ & 34782 & $0.18(0.01)$ & $0.91(0.001)$ & 1451 & $1.06(0.05)$ \\
\hline & $50-59$ & 138176 & 53.7 & 1923 & 1.73 & 25.57 & 14303 & $-0.01(0.01)$ & 16977 & $0.13(0.01)$ & $0.92(0.001)$ & 722 & $0.46(0.08)$ \\
\hline & $60-69$ & 41389 & 63.3 & 1916 & 1.71 & 25.33 & 5686 & $-0.08(0.02)$ & 4804 & $0.02(0.03)$ & $0.90(0.003)$ & 575 & $-0.31(0.10)$ \\
\hline & $70-79$ & 7679 & 72.6 & 1909 & 1.70 & 24.91 & 1542 & $0.10(0.03)$ & 1076 & $-0.44(0.05)$ & $0.88(0.007)$ & 105 & $-1.31(0.21)$ \\
\hline & $80-89$ & 506 & 82.0 & 1899 & 1.68 & 24.38 & 271 & $0.02(0.06)$ & 125 & $-0.73(0.16)$ & $0.86(0.023)$ & 0 & - \\
\hline \multirow[t]{8}{*}{ Female } & $15-19$ & 3647 & 17.2 & 1957 & 1.63 & 20.04 & 81 & $0.93(0.28)$ & 74 & $0.64(0.32)$ & $0.68(0.063)$ & 95 & $3.53(0.40)$ \\
\hline & $20-29$ & 29779 & 25.7 & 1953 & 1.63 & 21.82 & 1362 & $0.27(0.05)$ & 2333 & $0.77(0.05)$ & $0.84(0.006)$ & 1123 & $2.17(0.10)$ \\
\hline & $30-39$ & 73310 & 35.1 & 1944 & 1.62 & 23.09 & 2403 & $0.33(0.04)$ & 4398 & $0.96(0.03)$ & $0.87(0.004)$ & 1536 & $1.90(0.08)$ \\
\hline & $40-49$ & 103458 & 44.5 & 1937 & 1.61 & 24.56 & 5920 & $0.45(0.02)$ & 8215 & $0.94(0.02)$ & $0.91(0.002)$ & 1373 & $1.38(0.08)$ \\
\hline & $50-59$ & 82340 & 54.2 & 1929 & 1.60 & 25.34 & 8804 & $0.21(0.02)$ & 8317 & $0.65(0.02)$ & $0.91(0.002)$ & 790 & $0.56(0.10)$ \\
\hline & $60-69$ & 41391 & 63.3 & 1921 & 1.59 & 25.40 & 4884 & $0.08(0.02)$ & 4581 & $0.16(0.03)$ & $0.90(0.003)$ & 772 & $-0.79(0.11)$ \\
\hline & $70-79$ & 5046 & 72.7 & 1906 & 1.56 & 25.33 & 1855 & $-0.01(0.03)$ & 1412 & $-0.55(0.06)$ & $0.87(0.006)$ & 149 & $-2.08(0.28)$ \\
\hline & $80-89$ & 504 & 82.1 & 1897 & 1.54 & 24.40 & 242 & $0.02(0.09)$ & 131 & $-1.19(0.21)$ & $0.82(0.029)$ & 0 & - \\
\hline Both sex & 1 ages & 852824 & 46.0 & 1932 & - & 24.76 & 61559 & $0.14(0.01)$ & 95330 & $0.36(0.01)$ & $0.90(0.001)$ & 10578 & $1.16(0.03)$ \\
\hline
\end{tabular}

* Excluding 41752 with less than 5 years of follow-up, and standardising mean height and BMI for study

† For the 755890 participants with data on year of birth

¥ Self-correlation (within these age groups) between baseline BMI and BMI measured between 5-9 years of follow-up. The overall value of 0.90 is adjusted for the age/sex categories in this table. This value of 0.90 might suggest that the associations of risk with baseline BMI underestimate the associations with usual BMI by about $10 \%$, but this regression dilution was counterbalanced by a slight tendency of BMI values to disperse (webtable 4), so the underestimation was probably even less than $10 \%$, and, therefore, of little importance (particularly during the first decade of follow-up). 
Webtable 4: Change in BMI from baseline to a repeat measurement, by baseline BMI

\begin{tabular}{|c|c|c|c|c|c|c|c|c|}
\hline \multicolumn{3}{|c|}{$\begin{array}{l}\text { No. of people* at baseline, and their } \\
\text { mean BMI }\left(\mathrm{kg} / \mathrm{m}^{2}\right) \text { : }\end{array}$} & \multicolumn{6}{|c|}{$\begin{array}{l}\text { No. of people resurveyed, and their } \\
\text { change in BMI from baseline to first repeat measurement at: }\end{array}$} \\
\hline Baseline BMI & No. & Mean BMI & \multicolumn{2}{|c|}{ 0-4 (mean 2.6) years* } & \multicolumn{2}{|c|}{ 5-9 (mean 6.0) years } & \multicolumn{2}{|c|}{$\geq 10($ mean 13.1$)$ years } \\
\hline $15-20$ & 64703 & 18.91 & 3929 & $0.43(0.02)$ & 8844 & $0.62(0.02)$ & 888 & $2.02(0.08)$ \\
\hline $20-25$ & 427682 & 22.76 & 26800 & $0.26(0.01)$ & 48478 & $0.44(0.01)$ & 4942 & $1.59(0.04)$ \\
\hline $30-35$ & 58337 & 31.79 & 5986 & $-0.07(0.03)$ & 6275 & $0.24(0.03)$ & 974 & $0.02(0.11)$ \\
\hline $35-40$ & 11195 & 36.90 & 1460 & $-0.29(0.07)$ & 1388 & $-0.18(0.10)$ & 237 & $-0.89(0.29)$ \\
\hline $40-50$ & 3279 & 43.04 & 484 & $-0.45(0.15)$ & 451 & $-0.69(0.21)$ & 64 & $-4.51(0.70)$ \\
\hline Difference, 30-5 & 5-20†: & 14.17 & & $0.56(0.03)$ & & $0.50(0.04)$ & & $2.40(0.14)$ \\
\hline
\end{tabular}

* Excluding 41752 with less than 5 years of follow-up

$\dagger 30-50 \mathrm{~kg} / \mathrm{m}^{2}$ at baseline vs $15-20 \mathrm{~kg} / \mathrm{m}^{2}$ at baseline 
Webtable 5: Other cross-sectional associations* with BMI: height, cigarettes smoked per day, and grams of alcohol consumed per day

\begin{tabular}{|c|c|c|c|c|c|c|c|c|c|c|c|}
\hline & \multirow{3}{*}{$\begin{array}{c}\text { BMI } \\
\left(\mathrm{kg} / \mathrm{m}^{2}\right)\end{array}$} & \multicolumn{4}{|c|}{ All participants } & \multicolumn{3}{|c|}{ Current cigarette smokers $\dagger$} & \multicolumn{3}{|c|}{ Current alcohol drinkers $\ddagger$} \\
\hline & & \multirow{2}{*}{$\begin{array}{l}\text { No. of } \\
\text { people }\end{array}$} & \multirow{2}{*}{$\begin{array}{r}\text { Mean } \\
\text { BMI }\end{array}$} & \multicolumn{2}{|c|}{ Height } & \multirow{2}{*}{$\begin{array}{r}\text { No. of } \\
\text { smokers }\end{array}$} & \multicolumn{2}{|c|}{ Cigarettes/day§ } & \multirow{2}{*}{$\begin{array}{r}\text { No. of } \\
\text { drinkers }\end{array}$} & \multicolumn{2}{|c|}{ Alcohol g/day } \\
\hline & & & & Mean & SE & & Mean & SE & & Mean & SE \\
\hline \multirow[t]{10}{*}{ Male } & $15-17.5$ & 2454 & 16.8 & 1.73 & 0.0013 & 811 & 18.1 & 0.33 & 265 & 18.8 & 1.04 \\
\hline & $17.5-20$ & 26432 & 19.1 & 1.73 & 0.0004 & 8369 & 18.3 & 0.11 & 2651 & 16.8 & 0.33 \\
\hline & $20-22.5$ & 96693 & 21.4 & 1.73 & 0.0002 & 24930 & 18.6 & 0.06 & 14118 & 16.6 & 0.14 \\
\hline & $22.5-25$ & 168043 & 23.8 & 1.73 & 0.0002 & 36624 & 18.7 & 0.05 & 31528 & 16.6 & 0.10 \\
\hline & $25-27.5$ & 145249 & 26.1 & 1.73 & 0.0002 & 30013 & 19.0 & 0.05 & 29724 & 16.8 & 0.10 \\
\hline & $27.5-30$ & 65434 & 28.6 & 1.73 & 0.0003 & 13930 & 19.6 & 0.08 & 12846 & 17.7 & 0.15 \\
\hline & $30-32.5$ & 24891 & 31.0 & 1.72 & 0.0004 & 5105 & 19.7 & 0.13 & 5062 & 18.2 & 0.24 \\
\hline & $32.5-35$ & 8008 & 33.5 & 1.72 & 0.0007 & 1593 & 20.5 & 0.24 & 1800 & 18.7 & 0.40 \\
\hline & $\underline{35-50}$ & 4248 & 37.5 & 1.71 & 0.0010 & 843 & 20.6 & 0.33 & 1102 & 17.0 & 0.51 \\
\hline & All & 541452 & 24.9 & 1.73 & & 122218 & 18.9 & & 99096 & 17.0 & \\
\hline \multirow[t]{10}{*}{ Female } & $15-17.5$ & 3460 & 16.8 & 1.62 & 0.0010 & 514 & 15.8 & 0.38 & 358 & 8.5 & 0.51 \\
\hline & $17.5-20$ & 35673 & 19.1 & 1.62 & 0.0003 & 4212 & 15.2 & 0.13 & 4198 & 7.7 & 0.15 \\
\hline & $20-22.5$ & 90994 & 21.3 & 1.62 & 0.0002 & 11605 & 15.1 & 0.08 & 14208 & 7.7 & 0.08 \\
\hline & $22.5-25$ & 90033 & 23.7 & 1.62 & 0.0002 & 12009 & 15.1 & 0.08 & 15495 & 7.4 & 0.08 \\
\hline & $25-27.5$ & 59458 & 26.2 & 1.61 & 0.0002 & 7687 & 15.1 & 0.10 & 10558 & 7.0 & 0.09 \\
\hline & $27.5-30$ & 32545 & 28.7 & 1.61 & 0.0003 & 3936 & 15.1 & 0.14 & 5399 & 6.5 & 0.13 \\
\hline & $30-32.5$ & 19473 & 31.1 & 1.60 & 0.0004 & 2229 & 16.0 & 0.18 & 3151 & 6.3 & 0.17 \\
\hline & $32.5-35$ & 9979 & 33.6 & 1.60 & 0.0006 & 1140 & 15.8 & 0.18 & 1630 & 5.5 & 0.24 \\
\hline & $35-50$ & 11509 & 38.6 & 1.60 & 0.0006 & 1174 & 16.0 & 0.25 & 1807 & 5.3 & 0.23 \\
\hline & All & 353124 & 24.6 & 1.61 & & 44506 & 15.2 & & 56804 & 7.2 & \\
\hline
\end{tabular}

NB This table includes all participants irrespective of follow-up duration.

* At ages 35-89 years. Associations adjusted for age (7 groups) and study; association for alcohol also adjusted for smoking status (3 groups).

$\dagger$ Restricted to current smokers (cf. fig 1) in the 33 cohorts with information on cigarettes per day. Excludes smokers with values recorded as 0 cigarettes/day; $>60$ cigarettes/day taken as 60 cigarettes/day.

¥ Restricted to current regular drinkers (cf. fig 1) in the 21 cohorts with information on both current drinking status and alcohol grams/day. Excludes drinkers with values recorded as $0 \mathrm{~g} /$ day; $>100 \mathrm{~g}$ /day taken as $100 \mathrm{~g} /$ day.

$\S$ In the full BMI range $\left(15-50 \mathrm{~kg} / \mathrm{m}^{2}\right), 5 \mathrm{~kg} / \mathrm{m}^{2}$ lower BMI was associated with 0.5 fewer cigarettes per day per smoker (male $0.65 \mathrm{SE} 0.04$, female 0.18 SE 0.05), but — in a separate analysis of 2772 British smokers (not in the PSC) — with 11.8\% (SE 1.4) more cotinine per ml of blood among those smoking similar numbers of cigarettes (Parish S et al. BMJ 1995; 311: 471-77 \& personal communication). See also Istvan et al. Am J Epidemiol 2008; $139: 628-36$ and Campuzano et al. Nicotine Tob Res 2004; 6: 997-1008. 
Webtable 6: All-cause, vascular, diabetic, hepatic and renal mortality vs. baseline BMI in the ranges $15-25,25-50$ and (for selected endpoints) $15-50 \mathrm{~kg} / \mathrm{m}^{2}$ : hazard ratio (HR) per $5 \mathrm{~kg} / \mathrm{m}^{2}$ higher BMI; HR < 1 if BMI inversely associated with risk

\begin{tabular}{|c|c|c|c|c|c|}
\hline & \multicolumn{2}{|c|}{$15-25 \mathrm{~kg} / \mathrm{m}^{2}$} & \multicolumn{2}{|c|}{$25-50 \mathrm{~kg} / \mathrm{m}^{2}$} & \multirow{2}{*}{$\frac{15-50 \mathrm{~kg} / \mathrm{m}^{2}}{\mathrm{HR}(95 \% \mathrm{CI})}$} \\
\hline & Deaths & HR (95\% CI) & Deaths & HR (95\% CI) & \\
\hline ALL CAUSES & 35256 & $0.79(0.77-0.82)$ & 37493 & $1.29(1.27-1.32)$ & \\
\hline Male & 26720 & $0.79(0.76-0.82)$ & 27983 & $1.32(1.29-1.36)$ & \\
\hline Female & 8536 & $0.80(0.75-0.85)$ & 9510 & $1.26(1.23-1.30)$ & \\
\hline Never smoker & 7054 & $0.87(0.81-0.94)$ & 9849 & $1.32(1.28-1.36)$ & \\
\hline Current cigarette smoker & 19872 & $0.76(0.73-0.80)$ & 15777 & $1.25(1.21-1.29)$ & \\
\hline Other/unknown smoking at entry & 8330 & $0.81(0.75-0.87)$ & 11867 & $1.32(1.28-1.37)$ & \\
\hline Follow-up 0-4 years & 8158 & $0.62(0.58-0.66)$ & 7838 & $1.18(1.14-1.23)$ & \\
\hline Follow-up 5-9 years & 11319 & $0.70(0.66-0.75)$ & 11738 & $1.25(1.21-1.30)$ & \\
\hline Follow-up 10-14 years & 10763 & $0.81(0.76-0.87)$ & 11419 & $1.34(1.29-1.39)$ & \\
\hline Follow-up $\geq 15$ years & 13174 & $0.85(0.81-0.91)$ & 14336 & $1.30(1.25-1.34)$ & \\
\hline Cohorts of US health professionals* & 1257 & $1.02(0.78-1.33)$ & 1243 & $1.34(1.19-1.52)$ & \\
\hline ALL VASCULAR DISEASE & 13073 & $1.05(0.99-1.11)$ & 17343 & $1.41(1.37-1.45)$ & \\
\hline Male & 10204 & $1.09(1.02-1.17)$ & 13142 & $1.44(1.39-1.50)$ & \\
\hline Female & 2869 & $0.90(0.81-1.00)$ & 4201 & $1.36(1.31-1.42)$ & \\
\hline Never smoker & 2687 & $1.04(0.91-1.18)$ & 4668 & $1.43(1.36-1.49)$ & \\
\hline Current cigarette smoker & 7232 & $1.03(0.95-1.11)$ & 7210 & $1.34(1.28-1.41)$ & \\
\hline Other/unknown smoking at entry & 3154 & $1.11(0.98-1.26)$ & 5465 & $1.46(1.39-1.54)$ & \\
\hline Follow-up 0-4 years & 2689 & $0.94(0.83-1.06)$ & 3305 & $1.24(1.16-1.32)$ & \\
\hline Follow-up 5-9 years & 3988 & $0.93(0.84-1.04)$ & 5181 & $1.38(1.31-1.46)$ & \\
\hline Follow-up 10-14 years & 3893 & $1.12(1.00-1.26)$ & 5321 & $1.43(1.36-1.51)$ & \\
\hline Follow-up $\geq 15$ years & 5192 & $1.11(1.01-1.22)$ & 6841 & $1.41(1.35-1.47)$ & \\
\hline Ischaemic heart disease & 7461 & $1.22(1.13-1.32)^{*}$ & 10783 & $1.39(1.34-1.44)$ & $1.35(1.32-1.38) \dagger$ \\
\hline Male & 6070 & $1.27(1.16-1.39)$ & 8556 & $1.42(1.35-1.48)$ & \\
\hline Female & 1391 & $1.01(0.86-1.18)$ & 2227 & $1.35(1.28-1.43)$ & \\
\hline Never smoker & 1323 & $1.20(0.99-1.45)$ & 2651 & $1.41(1.33-1.50)$ & \\
\hline Current cigarette smoker & 4327 & $1.20(1.09-1.33)$ & 4690 & $1.33(1.25-1.41)$ & \\
\hline Other/unknown smoking at entry & 1811 & $1.26(1.07-1.50)$ & 3442 & $1.45(1.36-1.54)$ & \\
\hline Follow-up 0-4 years & 1480 & $1.16(0.98-1.38)$ & 2069 & $1.26(1.16-1.36)$ & \\
\hline Follow-up 5-9 years & 2281 & $1.03(0.89-1.20)$ & 3291 & $1.33(1.24-1.43)$ & \\
\hline Follow-up 10-14 years & 2323 & $1.42(1.21-1.66)$ & 3407 & $1.43(1.33-1.53)$ & \\
\hline Follow-up $\geq 15$ years & 2857 & $1.29(1.13-1.47)$ & 4085 & $1.42(1.34-1.51)$ & \\
\hline Stroke & 2964 & $0.92(0.82-1.03)$ & 3164 & $1.39(1.31-1.48)$ & \\
\hline Ischaemic stroke & 630 & $0.87(0.68-1.10)$ & 765 & $1.38(1.23-1.56)$ & \\
\hline Haemorrhagic stroke & 536 & $0.76(0.58-1.00)$ & 571 & $1.53(1.32-1.78)$ & \\
\hline Subarachnoid haemorrhage & 298 & $0.92(0.64-1.33)$ & 242 & $1.19(0.94-1.52)$ & \\
\hline Unclassified stroke & 1500 & $1.02(0.87-1.20)$ & 1586 & $1.40(1.28-1.53)$ & \\
\hline Other vascular disease $\$$ & 2648 & $0.84(0.75-0.95)$ & 3396 & $1.47(1.39-1.56)$ & \\
\hline Aortic aneurysm & 279 & $1.09(0.74-1.60)$ & 386 & $1.19(0.95-1.48)$ & $1.37(1.21-1.55)$ \\
\hline Pulmonary embolism & 113 & $0.88(0.50-1.54)$ & 145 & $1.24(0.90-1.70)$ & $1.14(0.94-1.38)$ \\
\hline Heart failure & 328 & $0.93(0.66-1.29)$ & 289 & $1.86(1.55-2.23)$ & \\
\hline Hypertensive disease & 227 & $1.17(0.77-1.76)$ & 327 & $2.03(1.75-2.36)$ & $1.71(1.53-1.91)$ \\
\hline Atherosclerosis \& other arterial dis. & 239 & $0.69(0.47-1.02)$ & 216 & $1.07(0.82-1.41)$ & $0.92(0.80-1.07)$ \\
\hline Inflammatory heart disease & 139 & $0.90(0.51-1.59)$ & 150 & $1.36(1.01-1.84)$ & $1.15(0.95-1.39)$ \\
\hline Rheumatic heart disease & 93 & $0.64(0.34-1.21)$ & 96 & $0.90(0.57-1.44)$ & $0.93(0.73-1.18)$ \\
\hline Sudden death & 199 & $0.95(0.57-1.57)$ & 281 & $1.44(1.13-1.84)$ & $1.35(1.16-1.57)$ \\
\hline Other heart disease (not IHD) & 664 & $0.72(0.57-0.91)$ & 807 & $1.51(1.34-1.70)$ & \\
\hline Other circulatory disease & 57 & $0.65(0.30-1.42)$ & 102 & $1.47(1.05-2.05)$ & $1.46(1.17-1.81)$ \\
\hline DIABETES & 171 & $0.96(0.59-1.55)$ & 393 & $2.16(1.89-2.46)$ & \\
\hline KIDNEY DISEASE (non-neoplastic) & 197 & $1.14(0.74-1.77)$ & 217 & $1.59(1.27-1.99)$ & $1.22(1.06-1.41)$ \\
\hline LIVER DISEASE (non-neoplastic) & 489 & $0.69(0.52-0.91)$ & 603 & $1.82(1.59-2.09)$ & \\
\hline Cirrhosis & 409 & $0.73(0.54-1.00)$ & 505 & $1.79(1.54-2.08)$ & \\
\hline
\end{tabular}

Analyses exclude the first 5 years of follow-up (unless otherwise indicated: italic), and are adjusted for study, sex, age at risk and baseline smoking status. Mean ages for IHD death and stroke death in various subgroups are given in figure 4, but the dependence of the HR on mean age at death is not corrected for either in that figure or in this webtable. HRs for the full range $\left(15-50 \mathrm{~kg} / \mathrm{m}^{2}\right)$ are not necessarily an average of the HRs for the two component ranges (cf. Simpson's paradox). For analyses restricted to those who had never smoked, see webtable 9

* Studies of US physicians, nurses or other health professionals (webtable 1), in which there may have been relatively little confounding by socioeconomic status.

$\dagger$ IHD HR (95\% CI): 1.27 (1.17-1.38) for $17.5-25 \mathrm{~kg} / \mathrm{m}^{2}$, and $1.36(1.33-1.39)$ for $17.5-50 \mathrm{~kg} / \mathrm{m}^{2}$ [1.31 (1.28-1.34) before adjustment for smoking status]

t Unspecified other vascular disease: 310 deaths at $15-25 \mathrm{~kg} / \mathrm{m}^{2}$ (HR $0.83,95 \%$ CI $\left.0.58-1.18\right), 597$ deaths at $25-50 \mathrm{~kg} / \mathrm{m}^{2}$ (HR 1.36, 95\% CI 1.20-1.55) 
Webtable 7: Neoplastic mortality vs. baseline BMI in the ranges 15-25, 25-50 and (for selected endpoints) $15-50 \mathrm{~kg} / \mathrm{m}^{2}$ : hazard ratio (HR) per $5 \mathrm{~kg} / \mathrm{m}^{2}$ higher BMI; HR $<1$ if BMI inversely associated with risk

\begin{tabular}{|c|c|c|c|c|c|}
\hline & \multicolumn{2}{|c|}{$15-25 \mathrm{~kg} / \mathrm{m}^{2}$} & \multicolumn{2}{|c|}{$25-50 \mathrm{~kg} / \mathrm{m}^{2}$} & \multirow{2}{*}{$\begin{array}{c}15-50 \mathrm{~kg} / \mathrm{m}^{2} \\
\text { HR }(95 \% \mathrm{CI})\end{array}$} \\
\hline & Deaths & HR (95\% CI) & Deaths & HR (95\% CI) & \\
\hline ALL NEOPLASTIC DISEASE & 11772 & $0.82(0.77-0.87)$ & 10820 & $1.10(1.06-1.15)$ & \\
\hline Male & 8824 & $0.78(0.73-0.84)$ & 8058 & $1.10(1.04-1.16)$ & \\
\hline Female & 2948 & $0.98(0.87-1.10)$ & 2762 & $1.11(1.05-1.18)$ & \\
\hline Never smoker & 2190 & $0.96(0.83-1.10)$ & 2704 & $1.13(1.05-1.21)$ & \\
\hline Current cigarette smoker & 6870 & $0.77(0.72-0.83)$ & 4637 & $1.06(0.99-1.13)$ & \\
\hline Other/unknown smoking at entry & 2712 & $0.88(0.77-1.00)$ & 3479 & $1.13(1.05-1.21)$ & \\
\hline Follow-up 0-4 years & 3164 & $0.64(0.57-0.71)$ & 2699 & $1.07(1.00-1.15)$ & \\
\hline Follow-up 5-9 years & 4078 & $0.77(0.69-0.85)$ & 3902 & $1.05(0.98-1.12)$ & \\
\hline Follow-up 10-14 years & 3713 & $0.79(0.70-0.88)$ & 3266 & $1.14(1.06-1.24)$ & \\
\hline Follow-up $\geq 15$ years & 3981 & $0.91(0.82-1.01)$ & 3652 & $1.13(1.05-1.21)$ & \\
\hline Lung cancer & 2959 & $0.71(0.63-0.79)$ & 2040 & $0.98(0.88-1.09)$ & $0.80(0.76-0.84)$ \\
\hline Upper aerodigestive cancer & 685 & $0.49(0.39-0.61)$ & 471 & $0.98(0.79-1.20)$ & $0.72(0.65-0.81)$ \\
\hline Oesophageal cancer & 339 & $0.52(0.38-0.72)$ & 259 & $0.93(0.69-1.24)$ & $0.76(0.65-0.88)$ \\
\hline Lung and upper aerodig. cancer* & 3644 & $0.66(0.59-0.73)$ & 2511 & $0.98(0.89-1.07)$ & \\
\hline Follow-up 0-4 years & 929 & $0.45(0.37-0.54)$ & 643 & $0.91(0.77-1.08)$ & \\
\hline Follow-up 5-9 years & 1299 & $0.58(0.48-0.69)$ & 988 & $1.01(0.87-1.17)$ & \\
\hline Follow-up 10-14 years & 1164 & $0.65(0.53-0.79)$ & 801 & $0.96(0.80-1.16)$ & \\
\hline Follow-up $\geq 15$ years & 1181 & $0.75(0.62-0.91)$ & 722 & $0.95(0.78-1.15)$ & \\
\hline Cancer, not lung or upper aerodig.* & 6134 & $0.94(0.87-1.02)$ & 6190 & $1.12(1.06-1.18)$ & \\
\hline Follow-up 0-4 years & 1879 & $0.78(0.68-0.90)$ & 1741 & $1.14(1.06-1.24)$ & \\
\hline Follow-up 5-9 years & 2311 & $0.88(0.76-1.02)$ & 2390 & $1.04(0.95-1.14)$ & \\
\hline Follow-up 10-14 years & 2042 & $0.91(0.78-1.07)$ & 1974 & $1.17(1.06-1.30)$ & \\
\hline Follow-up $\geq 15$ years & 1781 & $1.04(0.88-1.23)$ & 1826 & $1.17(1.06-1.29)$ & \\
\hline Liver cancer & 201 & $1.37(0.87-2.15)$ & 221 & $1.61(1.26-2.05)$ & $1.47(1.26-1.71)$ \\
\hline Kidney cancer & 193 & $1.02(0.64-1.64)$ & 254 & $1.30(1.02-1.67)$ & $1.23(1.06-1.43)$ \\
\hline Female breast cancer, age $<60 \uparrow$ & 291 & $1.51(1.01-2.25)$ & 171 & $1.10(0.88-1.39)$ & $1.15(1.02-1.31)$ \\
\hline Female breast cancer, age $\geq 60 \dagger$ & 192 & $1.06(0.66-1.70)$ & 228 & $1.11(0.91-1.36)$ & $1.15(1.02-1.31)$ \\
\hline Ovarian cancer & 182 & $0.74(0.47-1.18)$ & 158 & $0.98(0.75-1.28)$ & $1.00(0.85-1.16)$ \\
\hline Endometrial cancer & 35 & $0.97(0.32-2.94)$ & 67 & $1.18(0.80-1.74)$ & $1.38(1.08-1.77)$ \\
\hline Prostate cancer & 578 & $1.00(0.75-1.32)$ & 665 & $1.09(0.91-1.31)$ & $1.13(1.02-1.24)$ \\
\hline Pancreatic cancer & 470 & $0.87(0.65-1.17)$ & 520 & $1.04(0.86-1.25)$ & $1.07(0.97-1.19)$ \\
\hline Gallbladder cancer & 102 & $0.46(0.26-0.80)$ & 120 & $1.29(0.90-1.85)$ & $1.12(0.90-1.38)$ \\
\hline Stomach cancer & 934 & $0.86(0.70-1.05)$ & 651 & $1.11(0.94-1.32)$ & $0.98(0.90-1.07)$ \\
\hline Colorectal cancer & 1054 & $1.01(0.83-1.24)$ & 1200 & $1.20(1.07-1.35)$ & $1.20(1.12-1.28)$ \\
\hline Male & 782 & $0.99(0.77-1.26)$ & 931 & $1.32(1.14-1.52)$ & $1.29(1.19-1.40)$ \\
\hline Female & 272 & $1.08(0.74-1.57)$ & 269 & $1.04(0.86-1.26)$ & $1.05(0.94-1.18)$ \\
\hline Haematological cancer & 765 & $1.11(0.87-1.42)$ & 828 & $1.10(0.95-1.27)$ & $1.11(1.02-1.20)$ \\
\hline Leukaemia & 290 & $1.02(0.69-1.50)$ & 315 & $0.88(0.68-1.14)$ & $1.01(0.89-1.16)$ \\
\hline Non-Hodgkin's lymphoma & 193 & $1.19(0.70-2.02)$ & 196 & $1.09(0.81-1.47)$ & $1.13(0.96-1.34)$ \\
\hline Multiple myeloma & 138 & $1.38(0.77-2.48)$ & 190 & $1.34(1.01-1.77)$ & $1.27(1.07-1.50)$ \\
\hline Other specified cancer & 1137 & $0.87(0.71-1.06)$ & 1107 & $1.00(0.87-1.14)$ & $0.98(0.92-1.06)$ \\
\hline Unspecified cancer & 1994 & $0.83(0.72-0.96)$ & 2119 & $1.17(1.08-1.27)$ & \\
\hline
\end{tabular}

Analyses exclude the first 5 years of follow-up (unless otherwise indicated: italic), and are adjusted for study, sex, age at risk and baseline smoking status. HRs for the full range $\left(15-50 \mathrm{~kg} / \mathrm{m}^{2}\right)$ are not necessarily an average of the HRs for the two component ranges (cf. Simpson's paradox). For analyses restricted to those who had never smoked, see webtable 9.

* These are the cancer endpoints in figure 5; they do not include unspecified cancer.

$\uparrow$ By chance, the two age-specific breast cancer results for the range $15-50 \mathrm{~kg} / \mathrm{m}^{2}$ were the same to two decimal places.

t Difference between male and female HRs: $P$ for interaction $=0.08$ in the range $25-50 \mathrm{~kg} / \mathrm{m}^{2}, 0.002$ in the full range $15-50 \mathrm{~kg} / \mathrm{m}^{2}$. 
Webtable 8: Respiratory mortality, and remaining causes, vs. baseline BMI in the ranges 15-25 and $25-50 \mathrm{~kg} / \mathrm{m}^{2}$ : hazard ratio (HR) per $5 \mathrm{~kg} / \mathrm{m}^{2}$ higher BMI; HR $<1$ if BMI inversely associated with risk

\begin{tabular}{|c|c|c|c|c|}
\hline & \multicolumn{2}{|c|}{$15-25 \mathrm{~kg} / \mathrm{m}^{2}$} & \multicolumn{2}{|c|}{$25-50 \mathrm{~kg} / \mathrm{m}^{2}$} \\
\hline & Deaths & HR (95\% CI) & Deaths & HR (95\% CI) \\
\hline ALL RESPIRATORY DISEASE & 2426 & $0.31(0.28-0.35)$ & 1344 & $1.20(1.07-1.34)$ \\
\hline Male & 1993 & $0.31(0.27-0.35)$ & 1086 & $1.17(1.01-1.34)$ \\
\hline Female & 433 & $0.32(0.25-0.41)$ & 258 & $1.24(1.04-1.48)$ \\
\hline Never smoker & 370 & $0.48(0.36-0.65)$ & 257 & $1.16(0.93-1.45)$ \\
\hline Current cigarette smoker & 1497 & $0.29(0.25-0.34)$ & 645 & $1.26(1.07-1.48)$ \\
\hline Other/unknown smoking at entry & 559 & $0.26(0.20-0.34)$ & 442 & $1.15(0.93-1.40)$ \\
\hline Follow-up 0-4 years & 447 & $0.14(0.11-0.18)$ & 199 & $1.35(1.07-1.71)$ \\
\hline Follow-up 5-9 years & 713 & $0.25(0.20-0.31)$ & 375 & $1.08(0.85-1.37)$ \\
\hline Follow-up 10-14 years & 757 & $0.30(0.24-0.37)$ & 439 & $1.26(1.02-1.55)$ \\
\hline Follow-up $\geq 15$ years & 956 & $0.37(0.30-0.44)$ & 530 & $1.15(0.94-1.39)$ \\
\hline Chronic obstructive pulmonary disease & 1340 & $0.26(0.22-0.30)$ & 614 & $1.18(1.00-1.40)$ \\
\hline Follow-up 0-4 years & 255 & $0.11(0.08-0.16)$ & 87 & $1.12(0.74-1.69)$ \\
\hline Follow-up 5-9 years & 360 & $0.21(0.15-0.28)$ & 186 & $0.99(0.68-1.44)$ \\
\hline Follow-up 10-14 years & 409 & $0.22(0.16-0.29)$ & 187 & $1.25(0.90-1.74)$ \\
\hline Follow-up $\geq 15$ years & 571 & $0.31(0.24-0.40)$ & 241 & $1.24(0.93-1.65)$ \\
\hline Pneumonia & 695 & $0.46(0.37-0.57)$ & 482 & $1.15(0.96-1.38)$ \\
\hline Respiratory tuberculosis & 120 & $0.08(0.04-0.13)$ & 16 & - \\
\hline Excluding 10 years of follow-up & 72 & $0.09(0.04-0.19)$ & 7 & - \\
\hline Other respiratory disease & 271 & $0.48(0.34-0.69)$ & 232 & $1.34(1.04-1.72)$ \\
\hline OTHER SPECIFIED DISEASE* & 2049 & $0.62(0.54-0.71)$ & 1823 & $1.20(1.10-1.31)$ \\
\hline Male & 1536 & $0.60(0.51-0.70)$ & 1363 & $1.30(1.16-1.47)$ \\
\hline Female & 513 & $0.67(0.52-0.87)$ & 460 & $1.09(0.94-1.25)$ \\
\hline Never smoker & 532 & $0.62(0.47-0.81)$ & 523 & $1.31(1.13-1.52)$ \\
\hline Current cigarette smoker & 953 & $0.63(0.52-0.77)$ & 684 & $1.22(1.04-1.42)$ \\
\hline Other/unknown smoking at entry & 564 & $0.59(0.45-0.78)$ & 616 & $1.07(0.91-1.27)$ \\
\hline EXTERNAL CAUSE & 2112 & $0.82(0.71-0.95)$ & 1720 & $1.19(1.08-1.32)$ \\
\hline Male & 1641 & $0.88(0.74-1.04)$ & 1424 & $1.23(1.09-1.38)$ \\
\hline Female & 471 & $0.66(0.50-0.87)$ & 296 & $1.12(0.94-1.34)$ \\
\hline Never smoker & 506 & $0.84(0.62-1.13)$ & 510 & $1.19(1.01-1.41)$ \\
\hline Current cigarette smoker & 1128 & $0.78(0.65-0.95)$ & 695 & $1.21(1.03-1.43)$ \\
\hline Other/unknown smoking at entry & 478 & $0.86(0.63-1.18)$ & 515 & $1.17(0.97-1.41)$ \\
\hline UNKNOWN CAUSE & 2967 & $0.66(0.59-0.73)$ & 3230 & $1.24(1.17-1.32)$ \\
\hline Male & 1839 & $0.63(0.55-0.73)$ & 1988 & $1.27(1.16-1.40)$ \\
\hline Female & 1128 & $0.67(0.56-0.79)$ & 1242 & $1.22(1.12-1.32)$ \\
\hline Never smoker & 599 & $0.73(0.57-0.95)$ & 842 & $1.26(1.13-1.40)$ \\
\hline Current cigarette smoker & 1709 & $0.64(0.56-0.74)$ & 1410 & $1.20(1.08-1.32)$ \\
\hline Other/unknown smoking at entry & 659 & $0.62(0.48-0.80)$ & 978 & $1.28(1.15-1.43)$ \\
\hline
\end{tabular}

Analyses exclude the first 5 years of follow-up (unless otherwise indicated: italic), and are adjusted for study, sex, age at risk and baseline smoking status. For analyses restricted to those who had never smoked, see webtable 9 .

* Specified diseases other than vascular, neoplastic, respiratory, hepatic or renal diseases, and other than diabetes; see webtable 2. 
Webtable 9: Selected mortality in the ranges $15-25$ and $25-50 \mathrm{~kg} / \mathrm{m}^{2}$ among lifelong non-smokers: hazard ratio (HR) per $5 \mathrm{~kg} / \mathrm{m}^{2}$ higher BMI; HR < 1 if BMI inversely associated with risk

\begin{tabular}{|c|c|c|c|c|}
\hline & \multicolumn{2}{|c|}{$15-25 \mathrm{~kg} / \mathrm{m}^{2}$} & \multicolumn{2}{|c|}{$25-50 \mathrm{~kg} / \mathrm{m}^{2}$} \\
\hline ALL CAUSES & 7054 & $0.87(0.81-0.94)$ & 9849 & $1.32(1.28-1.36)$ \\
\hline Male* & 3694 & $0.87(0.78-0.97)$ & 4811 & $1.44(1.36-1.53)$ \\
\hline Female* & 3360 & $0.87(0.78-0.97)$ & 5038 & $1.27(1.22-1.32)$ \\
\hline Cohorts of US health professionals† & 450 & $1.30(0.82-2.05)$ & 418 & $1.21(0.96-1.54)$ \\
\hline ALL VASCULAR DISEASE & 2687 & $1.04(0.91-1.18)$ & 4668 & $1.43(1.36-1.49)$ \\
\hline Ischaemic heart disease $\neq$ & 1323 & $1.20(0.99-1.45)$ & 2651 & $1.41(1.33-1.50)$ \\
\hline Subarachnoid haemorrhage & 66 & $1.24(0.56-2.76)$ & 74 & $1.19(0.82-1.74)$ \\
\hline Unclassified stroke & 348 & $0.95(0.68-1.32)$ & 430 & $1.48(1.26-1.73)$ \\
\hline Other vascular disease & 613 & $0.85(0.66-1.09)$ & 955 & $1.52(1.38-1.67)$ \\
\hline DIABETES, LIVER OR KIDNEY DISEASE & 170 & $1.12(0.69-1.81)$ & 345 & $1.82(1.56-2.11)$ \\
\hline Diabetes $\ddagger$ & 39 & $2.46(0.75-8.01)$ & 135 & $1.88(1.50-2.36)$ \\
\hline Kidney disease (non-neoplastic) & 62 & $0.92(0.43-1.96)$ & 76 & $2.01(1.51-2.69)$ \\
\hline Liver disease (non-neoplastic) & 69 & $0.93(0.44-1.95)$ & 134 & $1.60(1.20-2.12)$ \\
\hline Cirrhosis & 52 & $0.96(0.40-2.31)$ & 107 & $1.63(1.18-2.25)$ \\
\hline ALL RESPIRATORY DISEASE & 370 & $0.48(0.36-0.65)$ & 257 & $1.16(0.93-1.45)$ \\
\hline Chronic obstructive pulmonary disease & 135 & $0.38(0.23-0.62)$ & 77 & $1.09(0.68-1.74)$ \\
\hline Follow-up $\geq 10$ years & 92 & $0.40(0.21-0.74)$ & 61 & $1.15(0.70-1.90)$ \\
\hline Pneumonia & 149 & $0.61(0.38-0.98)$ & 122 & $1.05(0.75-1.46)$ \\
\hline Respiratory tuberculosis & 29 & $0.10(0.04-0.29)$ & 2 & - \\
\hline Other respiratory disease & 57 & $1.13(0.48-2.70)$ & 56 & $1.49(1.00-2.22)$ \\
\hline OTHER SPECIFIED DISEASE & 532 & $0.62(0.47-0.81)$ & 523 & $1.31(1.13-1.52)$ \\
\hline EXTERNAL CAUSE & 506 & $0.84(0.62-1.13)$ & 510 & $1.19(1.01-1.41)$ \\
\hline UNKNOWN CAUSE & 599 & $0.73(0.57-0.95)$ & 842 & $1.26(1.13-1.40)$ \\
\hline
\end{tabular}

Analyses exclude the first 5 years (or 10 years: italic) of follow-up, and are adjusted for study, sex and age at risk.

* By chance, the all-cause $15-25 \mathrm{~kg} / \mathrm{m}^{2}$ results for the two sexes were the same to two decimal places.

† Studies of US physicians, nurses or other health professionals (webtable 1), in which there may have been relatively little confounding by socioeconomic status.

$\ddagger$ HR (95\% CI) for full BMI range (15-50 kg/m²): IHD 1.36 (1.30-1.42); diabetes 2.03 (1.72-2.41); lung cancer $0.99(0.81-1.22)$. 
Webtable 10: Assumed causal relative risks used to estimate* figure 7 survival curves

\begin{tabular}{|c|c|c|c|c|c|c|}
\hline \multirow[b]{2}{*}{ Cause of death } & \multirow{2}{*}{$\begin{array}{l}\text { Age at risk } \\
\text { (years) }\end{array}$} & \multicolumn{5}{|c|}{ Relative risk $\dagger$ for baseline BMI $\left(\mathrm{kg} / \mathrm{m}^{2}\right)$ range } \\
\hline & & 22.5-25 & $25-30$ & 30-35 & $35-40$ & $40-50$ \\
\hline \multirow[t]{3}{*}{ Ischaemic heart disease } & $35-59$ & 0.73 & 1 & 1.48 & 2.25 & 3.70 \\
\hline & $60-69$ & 0.83 & 1 & 1.47 & 1.94 & 2.92 \\
\hline & $70-79$ & 0.84 & 1 & 1.40 & 1.70 & 2.36 \\
\hline \multirow[t]{3}{*}{ Stroke } & $35-59$ & 0.95 & 1 & 1.72 & 3.10 & 6.19 \\
\hline & $60-69$ & 0.82 & 1 & 1.52 & 2.20 & 3.57 \\
\hline & $70-79$ & 0.95 & 1 & 1.35 & 1.75 & 2.47 \\
\hline \multirow[t]{3}{*}{ Other vascular disease } & $35-59$ & 0.89 & 1 & 1.83 & 2.81 & 5.30 \\
\hline & $60-69$ & 0.82 & 1 & 1.57 & 2.52 & 4.45 \\
\hline & $70-79$ & 0.82 & 1 & 1.28 & 1.88 & 2.78 \\
\hline Diabetes & $35-79$ & 0.58 & 1 & 2.57 & 4.90 & 13.05 \\
\hline Kidney disease (non-neoplastic) & $35-79$ & 1.13 & 1 & 1.88 & 2.71 & 5.02 \\
\hline Liver disease (non-neoplastic) & $35-79$ & 0.71 & 1 & 1.75 & 3.23 & 6.64 \\
\hline Liver and gallbladder cancer & $35-79$ & 0.74 & 1 & 2.05 & 2.43 & 4.19 \\
\hline Kidney cancer & $35-79$ & 0.80 & 1 & 1.34 & 1.64 & 2.21 \\
\hline Colorectal cancer, male $\ddagger$ & $35-79$ & 0.83 & 1 & 1.23 & 1.53 & $2.0 * *$ \\
\hline Colorectal cancer, female $\ddagger$ & $35-79$ & 0.91 & 1 & 1.21 & 1.24 & 1.33 \\
\hline Endometrial cancert\$ $\$$ & $35-79$ & 0.67 & 1 & 1.69 & 1.85 & 4.17 \\
\hline Female breast cancer, age $<60$ & $35-59$ & 1 & 1 & 1 & 1 & 1 \\
\hline Female breast cancer, age $\geq 60 \ddagger \|$ & $60-79$ & 0.75 & 1 & 1.22 & 1.27 & 1.58 \\
\hline Ovarian cancer: & $35-79$ & 0.87 & 1 & 1.01 & 1.31 & $1.5 * *$ \\
\hline Prostate cancer & $35-79$ & 0.89 & 1 & 1.12 & 1.23 & 1.39 \\
\hline Pancreatic cancer & $35-79$ & 0.85 & 1 & 1.12 & 1.17 & 1.28 \\
\hline Lung \& upper aerodig. cancer $\dagger \dagger$ & $35-79$ & $(1.11)$ & 1 & $(0.96)$ & $(0.92)$ & $(0.88)$ \\
\hline Other specified cancer $\dagger \dagger$ & $35-79$ & $(0.99)$ & 1 & $(1.03)$ & $(1.08)$ & (1.13) \\
\hline Unspecified cancer & - & - & - & - & - & 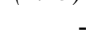 \\
\hline Respiratory disease $\dagger \dagger$ & $35-79$ & $(1.09)$ & 1 & $(1.10)$ & $(1.51)$ & (1.95) \\
\hline Other specified disease $\dagger \dagger$ & $35-79$ & $(1.02)$ & 1 & $(1.24)$ & $(1.72)$ & $(2.36)$ \\
\hline External cause $\dagger \dagger$ & $35-79$ & $(1.01)$ & 1 & $(1.18)$ & $(1.44)$ & $(1.28)$ \\
\hline Unknown cause & - & - & - & - & - & - \\
\hline
\end{tabular}

* The causal relative risks were applied to EU 2000 cause-specific death rates at ages 35-39 to 75-79 (with summation yielding the all-cause rates). For older ages, the BMI-specific relative risks for all-cause mortality at ages $75-79$ (relative to BMI $25-30 \mathrm{~kg} / \mathrm{m}^{2}$ ) were applied to estimates of the all-cause death rates for single years of age in England and Wales in 2000. (As a sensitivity analysis, the square roots of these relative risks yielded similar results.)

$\dagger$ Relative risks (RR) are from the PSC unless indicated otherwise. These RRs were adjusted for study, age at risk, sex and baseline smoking, and the first 5 years of follow-up were excluded. The RRs for vascular disease were assumed to depend on age but not, given age, on sex. (The lower RRs for women than for men in figure 4 chiefly reflect the difference between the sexes in mean age at death.) RRs in italics were, to improve statistical stability, calculated from regressions on BMI as a continuous variable in the range $25-50 \mathrm{~kg} / \mathrm{m}^{2}$ (or, for prostate and pancreatic cancers, $\left.15-50 \mathrm{~kg} / \mathrm{m}^{2}\right)$.

\$ These RRs are, for greater statistical stability, from the Cancer Prevention Study-II (CPS-II), ${ }^{4}$ and were adjusted for age, smoking, physical activity and education (among other variables). Mean age at baseline in CPS-II was 57 years, and deaths were recorded during follow-up for 16 years. Analyses did not exclude early follow-up, but did exclude anyone with a history of cancer or recent notable weight loss. CPS-II RRs for the $18.5-24.9 \mathrm{~kg} / \mathrm{m}^{2}$ category have been inserted above into the $22.5-25 \mathrm{~kg} / \mathrm{m}^{2}$ column.

$\S$ In CPS-II but not PSC, endometrial cancer included ICD-9 179 (uterus, part unspecified).

|| Defined in CPS-II as postmenopausal breast cancer.

** RR unavailable in CPS-II, but estimated here from the trend in RRs across lower BMI groups

† For these causes of death the RRs describing the associations of BMI with risk in the PSC are given in parentheses, as it was assumed in estimating the effects of BMI on mortality for figure 7 that these associations could have been non-causal (ie, these RRs were taken as 1.0 in calculating figure 7). Sensitivity analyses that instead used the RRs in parentheses showed that this assumption did not materially alter the median survival in any BMI category. 
Webtable 11: Cumulative mortality at ages 35-79 implied by webtable 10: male

\begin{tabular}{|c|c|c|c|c|c|c|}
\hline \multirow[b]{2}{*}{ Cause of death } & \multirow{2}{*}{$\begin{array}{l}\text { Age at risk } \\
\text { (years) }\end{array}$} & \multicolumn{5}{|c|}{$\begin{array}{c}\text { Cumulative mortality* }(\%) \text { for } \\
\text { baseline BMI }\left(\mathrm{kg} / \mathrm{m}^{2}\right) \text { range }\end{array}$} \\
\hline & & $22.5-25$ & $25-30$ & $30-35$ & $35-40$ & $40-50$ \\
\hline \multirow[t]{3}{*}{ Ischaemic heart disease } & $35-59$ & 1.1 & 1.6 & 2.3 & 3.5 & 5.8 \\
\hline & $60-69$ & 2.7 & 3.3 & 4.8 & 6.3 & 9.5 \\
\hline & $70-79$ & 7.4 & 8.8 & 12.3 & 14.9 & 20.7 \\
\hline \multirow[t]{3}{*}{ Stroke } & $35-59$ & 0.4 & 0.4 & 0.7 & 1.3 & 2.6 \\
\hline & $60-69$ & 0.9 & 1.0 & 1.6 & 2.3 & 3.7 \\
\hline & $70-79$ & 4.0 & 4.3 & 5.8 & 7.5 & 10.5 \\
\hline \multirow[t]{3}{*}{ Other vascular disease } & $35-59$ & 0.7 & 0.8 & 1.5 & 2.3 & 4.4 \\
\hline & $60-69$ & 1.4 & 1.7 & 2.7 & 4.4 & 7.8 \\
\hline & $70-79$ & 4.6 & 5.7 & 7.2 & 10.7 & 15.7 \\
\hline ALL VASCULAR & $35-79$ & 23.3 & 27.6 & 39.0 & 53.2 & 80.8 \\
\hline Diabetes & $35-79$ & 0.9 & 1.5 & 3.8 & 7.3 & 19.5 \\
\hline Kidney disease (non-neoplastic) & $35-79$ & 0.7 & 0.6 & 1.2 & 1.7 & 3.2 \\
\hline Liver disease (non-neoplastic) & $35-79$ & 1.5 & 2.1 & 3.7 & 6.8 & 14.0 \\
\hline ALL HEPATO-RENAL-DIABETIC & $35-79$ & 3.1 & 4.3 & 8.7 & 15.9 & 36.8 \\
\hline Liver and gallbladder cancer & $35-79$ & 0.9 & 1.2 & 2.5 & 2.9 & 5.1 \\
\hline Kidney cancer & $35-79$ & 0.6 & 0.8 & 1.0 & 1.2 & 1.7 \\
\hline Colorectal cancer, male & $35-79$ & 2.5 & 3.1 & 3.7 & 4.7 & 6.1 \\
\hline Prostate cancer & $35-79$ & 2.2 & 2.4 & 2.7 & 3.0 & 3.4 \\
\hline Pancreatic cancer & $35-79$ & 1.1 & 1.3 & 1.5 & 1.5 & 1.7 \\
\hline Lung cancer & $35-79$ & 10.5 & 10.5 & 10.5 & 10.5 & 10.5 \\
\hline Other specified cancer & $35-79$ & 7.5 & 7.5 & 7.5 & 7.5 & 7.5 \\
\hline Unspecified cancer† & $35-79$ & 0.0 & 0.0 & 0.0 & 0.0 & 0.0 \\
\hline ALL NEOPLASTIC & $35-79$ & 25.3 & 26.8 & 29.4 & 31.4 & 35.9 \\
\hline Respiratory disease & $35-79$ & 6.7 & 6.7 & 6.7 & 6.7 & 6.7 \\
\hline Other specified disease & $35-79$ & 6.7 & 6.7 & 6.7 & 6.7 & 6.7 \\
\hline External cause & $35-79$ & 3.4 & 3.4 & 3.4 & 3.4 & 3.4 \\
\hline Unknown cause $†$ & $35-79$ & 0.0 & 0.0 & 0.0 & 0.0 & 0.0 \\
\hline $\begin{array}{l}\text { ALL CAUSES (and corresponding } \ddagger \\
\text { risk of } 35 \text { year old dying before } 80 \text { ) }\end{array}$ & $35-79$ & $\begin{array}{r}68.6 \\
(50 \%)\end{array}$ & $\begin{array}{r}75.4 \\
(53 \%)\end{array}$ & $\begin{array}{r}93.9 \\
(61 \%)\end{array}$ & $\begin{array}{l}117.3 \\
(69 \%)\end{array}$ & $\begin{array}{l}170.3 \\
(82 \%)\end{array}$ \\
\hline
\end{tabular}

* For the BMI $25-30 \mathrm{~kg} / \mathrm{m}^{2}$ category, the cumulative mortality in a given range of ages at risk is assumed to be the total duration in years (eg, 25 for the range 35-59) times the average of the annual death rates in each of the 5-year age ranges within it (eg, 35-39, 40-44 etc). The death rates are those for the European Union in 2000 (ie, the aggregate of 15 western European countries). For the other BMI categories, the cumulative mortality is assumed to be that at BMI $25-30 \mathrm{~kg} / \mathrm{m}^{2}$ multiplied by the corresponding relative risk in webtable 10 (unless the relative risk is in parentheses, in which case the multiplier is 1). Cumulative mortalities in different age ranges, or from different causes of death, are exactly additive.

† Unspecified cancer deaths were redistributed over the other neoplastic categories by dividing each category's rate by 1 minus the proportion of neoplastic deaths that were unspecified (ie, by 0.94 ). Deaths from unknown cause were then redistributed over all other mortality categories (including the neoplastic categories) by dividing each category's rate by 1 minus the proportion of all deaths that had an unknown cause (ie, by 0.98 ).

$\ddagger$ If the cumulative mortality at ages $35-79$ is $c \%$ then the probability that a 35-year-old will die before age 80 is 1 - $\exp (-c / 100)$. For example, if $c=75.4$ then the survival probability is $1-\exp (-0.754)=0.53$. 
Webtable 12: Cumulative mortality at ages 35-79 implied by webtable 10: female

\begin{tabular}{|c|c|c|c|c|c|c|}
\hline \multirow[b]{2}{*}{ Cause of death } & \multirow{2}{*}{$\begin{array}{l}\text { Age at risk } \\
\text { (years) }\end{array}$} & \multicolumn{5}{|c|}{$\begin{array}{l}\text { Cumulative mortality* }(\%) \text { for } \\
\text { baseline BMI }\left(\mathrm{kg} / \mathrm{m}^{2}\right) \text { range }\end{array}$} \\
\hline & & 22.5-25 & $25-30$ & $30-35$ & $35-40$ & $40-50$ \\
\hline \multirow[t]{3}{*}{ Ischaemic heart disease } & $35-59$ & 0.2 & 0.3 & 0.5 & 0.7 & 1.1 \\
\hline & $60-69$ & 0.8 & 1.0 & 1.5 & 2.0 & 2.9 \\
\hline & $70-79$ & 3.5 & 4.1 & 5.8 & 7.0 & 9.8 \\
\hline \multirow[t]{3}{*}{ Stroke } & $35-59$ & 0.3 & 0.3 & 0.5 & 0.8 & 1.6 \\
\hline & $60-69$ & 0.5 & 0.6 & 0.9 & 1.3 & 2.1 \\
\hline & $70-79$ & 2.9 & 3.0 & 4.1 & 5.3 & 7.5 \\
\hline \multirow[t]{3}{*}{ Other vascular disease } & $35-59$ & 0.3 & 0.3 & 0.6 & 1.0 & 1.8 \\
\hline & $60-69$ & 0.7 & 0.8 & 1.3 & 2.1 & 3.7 \\
\hline & $70-79$ & 3.0 & 3.7 & 4.7 & 6.9 & 10.2 \\
\hline ALL VASCULAR & $35-79$ & 12.1 & 14.2 & 19.8 & 27.1 & 40.8 \\
\hline Diabetes & $35-79$ & 0.7 & 1.1 & 2.9 & 5.5 & 14.7 \\
\hline Kidney disease (non-neoplastic) & $35-79$ & 0.4 & 0.4 & 0.7 & 1.0 & 1.9 \\
\hline Liver disease (non-neoplastic) & $35-79$ & 0.7 & 0.9 & 1.6 & 3.0 & 6.2 \\
\hline ALL HEPATO-RENAL-DIABETIC & $35-79$ & 1.8 & 2.5 & 5.3 & 9.6 & 22.9 \\
\hline Liver and gallbladder cancer & $35-79$ & 0.4 & 0.6 & 1.1 & 1.3 & 2.3 \\
\hline Kidney cancer & $35-79$ & 0.3 & 0.3 & 0.4 & 0.5 & 0.7 \\
\hline Colorectal cancer, female & $35-79$ & 1.6 & 1.8 & 2.1 & 2.2 & 3.5 \\
\hline Endometrial cancer & $35-79$ & 0.1 & 0.2 & 0.4 & 0.4 & 0.4 \\
\hline Female breast cancer, age $<60$ & $35-59$ & 0.9 & 0.9 & 0.9 & 0.9 & 0.9 \\
\hline Female breast cancer, age $\geq 60$ & $60-79$ & 1.5 & 2.0 & 2.4 & 2.5 & 4.0 \\
\hline Ovarian cancer & $35-79$ & 0.8 & 1.0 & 1.0 & 1.3 & 1.9 \\
\hline Pancreatic cancer & $35-79$ & 0.8 & 0.9 & 1.0 & 1.1 & 1.2 \\
\hline Lung cancer & $35-79$ & 2.4 & 2.4 & 2.4 & 2.4 & 2.4 \\
\hline Other specified cancer & $35-79$ & 4.3 & 4.3 & 4.3 & 4.3 & 4.3 \\
\hline Unspecified cancer $\dagger$ & $35-79$ & 0.0 & 0.0 & 0.0 & 0.0 & 0.0 \\
\hline ALL NEOPLASTIC & $35-79$ & 13.1 & 14.4 & 16.1 & 16.9 & 21.7 \\
\hline Respiratory disease & $35-79$ & 2.9 & 2.9 & 2.9 & 2.9 & 2.9 \\
\hline Other specified disease & $35-79$ & 4.5 & 4.5 & 4.5 & 4.5 & 4.5 \\
\hline External cause & $35-79$ & 1.4 & 1.4 & 1.4 & 1.4 & 1.4 \\
\hline Unknown cause $\dagger$ & $35-79$ & 0.0 & 0.0 & 0.0 & 0.0 & 0.0 \\
\hline $\begin{array}{l}\text { ALL CAUSES (and corresponding } \$ \\
\text { risk of } 35 \text { year old dying before } 80 \text { ) }\end{array}$ & $35-79$ & $\begin{array}{r}35.8 \\
(30 \%)\end{array}$ & $\begin{array}{r}39.7 \\
(33 \%)\end{array}$ & $\begin{array}{r}49.9 \\
(39 \%)\end{array}$ & $\begin{array}{r}62.4 \\
(46 \%)\end{array}$ & $\begin{array}{r}94.2 \\
(61 \%)\end{array}$ \\
\hline
\end{tabular}

Footnotes as in webtable 11. 